Managing the terror of publication bias: A comprehensive $p$-curve analysis of the Terror Management Theory literature

Lihan Chen ${ }^{1}$, Rachele Benjamin ${ }^{1}$, Addison Lai, \& Steven J. Heine University of British Columbia

January 4, 2022, correction to the preprint: In previous versions, the total included studies was reported as $\mathrm{K}=860$. The $p$-curve automatically excluded 42 of those studies, whose re-computed $p$-values exceeded $p=.05$. We corrected the number of included studies to $\mathrm{K}=818$. We thank Dr. Uli Schimmack for sharing his Z-curve re-analysis, which allowed us to catch our mistake. The current version of this article has been corrected.

${ }^{1}$ The first two authors contributed equally to the manuscript 


\begin{abstract}
A key prediction of Terror Management Theory is that people affirm their cultural worldview after they are reminded of death. This mortality salience (MS) hypothesis has been widely explored, yet the presence of questionable research practices may impact the replicability of this literature. We assess the evidential value of the MS hypothesis by conducting a pre-registered $p$ curve analysis of 818 published studies. Our results suggest that there are nonzero effects in this literature and that power is larger for studies conducted with multiple delays between the independent and dependent variables, for studies that test for main effects in comparison to those that test for interactions, and for studies conducted more recently. However, since the estimated average power of MS studies is $26 \%$, direct replications are unlikely to succeed. We recommend researchers consider our evidence when planning their samples, and that they anticipate smaller effects by increasing their sample sizes.
\end{abstract}

Keywords: Terror management theory, $p$-curve, meta-analysis, replication crisis 


\section{Managing the terror of publication bias: A comprehensive $p$-curve analysis of the Terror Management Theory literature.}

Terror Management Theory (TMT) begins with a simple premise: humans, like all species, have a fundamental need to survive, yet they are aware that they are ultimately going to fail at this quest, which produces existential terror (Greenberg, Pyszczynski, \& Solomon, 1986). Building on the work of Ernest Becker (1973) the theory suggests that people seek to dispel their terror by pursuing feelings of symbolic immortality; that is, because one's culture can exist long after one's individual death, people can become imbued with the potential immortality that is inherent in their cultures by either bolstering their cultural worldview or by viewing themselves as living up to the standards of their cultures. People can achieve feelings of symbolic immortality by engaging in a variety of symbolic acts, such as investing in one's national identity (Greenberg, Pyszczynski, Solomon, Simon, \& Breus, 1994), donating to charity (Dunne, Gallagher, \& Matthews, 2015; Jonas, Schimel, Greenberg, \& Pyszczynski, 2002), derogating members of other religious groups (Vail, Courtney, \& Arndt, 2019), or punishing people who are breaking the laws (Rosenblatt, Greenberg, Solomon, Pyszczynski, \& Lyon, 1989).

TMT offers a variety of predictions about how people behave when confronted with reminders of their own mortality. The most widely studied hypothesis is the mortality salience hypothesis (MS hypothesis), which predicts that when people consider their inevitable demise, they are more eager to defend their cultural worldview (Rosenblatt et al., 1989). Whereas TMT suggests that people are constantly engaged at keeping their mortality concerns at bay, the MS hypothesis proposes that people have particular reactions when such thoughts seep into awareness: Specifically, acute reminders of death are thought to increase people's need to bolster their cultural worldview, their self-esteem, and their attachments. Moreover, the MS hypothesis 
posits a distal defense against death reminders, which involves the unconscious process of engaging in worldview defense and self-esteem maintenance (Solomon, Greenberg, \& Pyszczynski, 1991), rather than engaging in proximal defenses, which involves confronting death thoughts head-on.

The MS hypothesis is by far the most generative idea from TMT (Pyszczynski, Solomon, \& Greenberg, 2015), as it has been explored in more than 1500 studies conducted in more than 20 countries (Vail et al., 2019), and, several of the foundational studies have been cited over 1000 times each (see Greenberg et al., 1986; Greenberg et al., 1994). The results of this expansive literature have been meta-analyzed several times (see Burke, Martens, \& Faucher, 2010; Burke, Kosloff, \& Landau, 2013; Martens, Burke, Schimel, \& Faucher, 2011), pointing to a well-studied robust moderate effect, which lends itself nicely to replication.

Many Labs 4 (Klein et al., 2019) attempted to replicate one of the early experiments on the MS hypothesis (Greenberg et al., 1994, Study 1), across 21 labs and 2,220 participants. In a blow to the MS hypothesis, the meta-analytic effect size was negligible (Hedges' $g=0.03$ ), even in those studies where the replicators strictly adhered to the original authors guidelines. One criticism of the Many Labs 4 replication was that it included studies with small samples that did not meet the pre-registered sample size requirement (Chatard, Hirschberger, \& Pyszczynski, 2020). Yet the re-analysis in Chatard et al. (2020) that excluded the studies with smaller samples only found that the key effect was replicated in the studies that followed the advice of the original authors. The disappointing findings of this replication effort appears to have sparked more scrutiny of the overall state of the TMT literature, and has elicited questions regarding whether many of the positive test results have appropriately adhered to the fields emerging standards of replicability-planning power, pre-registering methods and hypotheses, and 
disclosing how many of these studies are in the file drawer (Giofrè, Cumming, Fresc, Boedker, \& Tressoldi, 2017; Wicherts et al., 2016).

A number of other recent pre-registered studies of the MS hypothesis have also failed to replicate (see Rodríguez-Ferreiro, Barberia, González-Guerra, \& Vadillo, 2019; Sætrevik \& Sjåstad, 2019; Schindler, Reinhardt, \& Reinhard, 2021). In some cases, null results have emerged despite more careful power planning and close adherence to the pre-registration (see Schindler et al., 2021) and despite efforts to adapt methods so they are appropriate to the cultural context of their sample (see Sætrevik \& Sjåstad, 2019). On the other hand, there are also counterexamples of successful pre-registered experiments (Dunn, White, \& Dahl, 2020; Schindler, Pfattheicher, Reinhard, \& Greenberg, 2019; Vail, Courtney, \& Arndt, 2019) but the search for the ideal study conditions continues.

\section{Searching for the Ideal Conditions to Test the MS Hypothesis}

One possible reason for the conflicting findings of these replication efforts is that the MS hypothesis may be more reliably tested in some studies than in others. For example, there may be some individuals who are more reactive to reminders of their own mortality; some behaviors may be more sensitive tests of the hypothesis than others; and, some studies may be designed such that it is more straightforward to detect how thoughts of mortality affect other thoughts and behaviors. Below we summarize some moderating variables of the MS hypothesis that have been identified in past empirical research and through attention to the theoretical tenets of TMT.

\section{Larger delays between independent and dependent variables}

Early TMT research found that reminding people of death elicits a different kind of proximal response when the dependent measure is completed immediately after the reminder, leading people to rationalize or attempt to comprehend the threat (Greenberg et al., 1994). In 
contrast, the MS hypothesis is concerned with distal responses to thoughts of death-that is, the efforts that people make to keep mortality thoughts outside of their conscious awareness (Greenberg et al., 1994). To elicit distal responses, researchers typically add delay tasks between the independent and dependent variables, and meta-analytic evidence suggests that longer delays elicit stronger effects on the dependent measure (Burke et al., 2010). This empirical finding aligns well with the theory of proximal and distal responding: The longer the opportunity that participants have to engage in strategies to keep thoughts about their mortality at bay, the stronger the evidence is for the MS hypothesis.

\section{Focus on undergraduates}

Researchers report that older adults and seniors are less reactive to reminders of their mortality than are undergraduates (Chopik, 2017; Cicirelli, 2001, 2002; Fortner \& Neimeyer, 1999). Though death may feel closer for older individuals, their capacity to cope with their impending mortality appears to improve, either because they are more practiced at contemplating their inevitable demise or because they have achieved more goals that bring them closer to fulfillment (Fortner \& Neimeyer, 1999). Though many studies show attenuated reactions to death reminders (see Burke et al., 2010) some find that older adults respond differently but not less: Rather than negative worldview-restoring behaviors, like derogating an outgroup member, in the face of death awareness older adults may approach more generative worldviews like those targeting self-improvement or improving the world around them (Greenberg \& Helm, 2019; Maxfield et al., 2014; Maxfield, Pyszczynski, Greenberg, Pepin, \& Davis, 2012; Maxfield et al., 2007). Most MS studies have relied on undergraduate samples (Greenberg \& Helm, 2019) but in addition to being a convenient sample to obtain, undergraduates may have stronger and more typical responses to death reminders. 


\section{A focus on US samples}

Another moderator may be the culture of one's sample. It is conceivable that some cultures have developed more effective strategies for coping with death thoughts. Indeed, Burke et al. (2010) identified that effect sizes for tests of the MS hypothesis were larger in American samples than they were in other Western samples, which were in turn larger than those found in some non-Western samples.

There are a few reasons why the MS hypothesis may be more true of Americans than other cultural groups. First, mortality salience may be more threatening depending on one's cultural context (Park \& Pyszczynski, 2016; Schumaker, Barraclough, \& Vagg, 1988). Americans may have larger reactions to thoughts about death as they are among the most individualistic people living on the planet (e.g., Hofstede, 2001; Lipset, 1996; Oyserman, Coon, \& Kemmelmeier, 2002); perhaps when people's lives are focused more on their individual selves than on their network of relationships the idea of death is more annihilating (Park \& Pyszczynski, 2015; Pyszczynski et al., 2015). It is also possible that different religions may offer more useful coping mechanisms for thoughts of death. Park and Choi (2002) found that reminding Korean college students of Taoist ideas made them less reactive to a mortality reminder. In another study, compared to non-Buddhists, Korean Buddhists were less likely to derogate targets who criticized Korea, whose responses were mitigated by a 30-minute Zen meditation session (Park \& Pyszczynski, 2015).

Second, research finds that some non-Western samples are less motivated to maintain high self-esteem (Heine, Lehman, Markus, \& Kitayama, 1999). Consequently, they may have less to gain from responding to death reminders with worldview-affirming behaviors and cognitions (Greenberg et al., 1986; Heine, Harihara, \& Niiya, 2002; Park \& Pyszczynski, 2016). 
If death reminders lead to desires to enhance self-esteem, then people from cultures where selfesteem is less prioritized might be less likely to attempt to restore self-esteem in the face of their mortality thoughts.

Last, culture might affect not just the degree, but the type of defensive responses people have to death reminders. For example, Yen and Cheng (2010) studying Taiwanese participants found that death reminders did not elicit pro-Taiwanese bias (the typical reaction to MS) but did increase participants' tendencies to embrace fatalism - a cultural orientation more common among Taiwanese. Hence, it seems important to assess whether evidence for the MS hypothesis emerges differently across cultural contexts.

\section{Challenging the ideal conditions}

The number of delays, participants' age, and participants' culture, are three moderators derived from the logic of terror management theory, and they may account for why people's worldview defense takes different forms. It is possible that an inattention to these moderators are responsible for some of the failed replications of the MS hypothesis noted earlier; however, this seems less likely as some recent studies failed to replicate some foundational effects, despite that the researchers planned around potential hidden moderators (see Klein et al., 2019; RodríguezFerreiro et al., 2019; Sætrevik \& Sjåstad, 2019; Schindler et al., 2021). Moreover, given the debate about the replicability of the MS hypothesis it is quite possible that the replicability of these moderators is also in question.

\section{The Replication Crisis}

In recent years, the replicability of several high profile theories in psychology has been called into question (Open Science Collaboration, 2015; Vohs et al., 2021; Wagenmakers et al., 2016), and many have argued that the field is caught in a replication crisis (e.g., Pashler \& 
Wagenmakers, 2012). Since the widely circulated article by Ioannidis (2005) called attention to the problems of publication bias and questionable research practices (QRPs), psychologists have become increasingly wary of the results from past psychological research. Before reporting standards in psychology changed to emphasize replicability (Giofrè et al., 2017; Nosek et al., 2015), psychologists frequently engaged in various QRPs that inflated effect size estimates (John, Loewenstein, \& Prelec, 2012; Simmons, Nelson, \& Simonsohn, 2011), such as hypothesizing after the results are known (also known as HARKing; Kerr, 1998); leaving moderators, dependent, and independent variables undisclosed if they did not produce an effect (Simmons et al., 2011), adding participants in increments until an effect was significant, and selectively excluding outliers in order to report $p<.05$ (John et al., 2012). These strategies are collectively known as p-hacking (Simonsohn, Nelson, \& Simmons, 2014a), and though they are not always maliciously done, they overestimate the true size of a given effect. If these actions can change the outcome of individual studies, they may have wider implications for the field of psychology as a whole.

An increasing awareness of the problems of $p$-hacking has led psychologists to question some key assumptions that had earlier guided the field (e.g., Simonsohn et al., 2014a). One of these is that if a literature was large, and empirical evidence supporting an effect had been established many times, the effect must be true. Though it may seem counterintuitive, some wellestablished phenomena — apparently demonstrated in hundreds of studies — have been found to not replicate (e.g., Open Science Collaboration, 2015; Vohs et al., 2021; Wagenmakers et al., 2016). Fergus and Valentiner (2012) proposed that large theories may fail to replicate, not in spite of their prominence, but because of it. That is, theories may survive failed replications because any one study cannot undermine the wealth of replications in the published literature. 
Though an effect may appear robust, it is unlikely that the bulk of the studies in a literature represent true replications of it as they are often indirect extensions of the original finding. And the field's aversion to accepting the null hypothesis is such that failures to replicate may cause the researcher to second-guess their results, motivating them to $p$-hack, or leave the null findings in the file drawer. These kinds of practices prevent theory falsification but maintain the illusion that a finding is robust (Fergus \& Valentiner, 2012).

Is it possible that the effect of death reminders on cultural worldview defense is an example of a non-replicating phenomenon? Critics have found at least three reasons to be skeptical of the MS hypothesis. First, the many studies in the literature are highly heterogeneous, and direct replications have not been the norm. For example, while worldview affirmation is the dependent variable in most MS studies, affirmation has been operationalized by a large number of distinct behaviours, for example, from punishing a moral violator (Rosenblatt et al., 1989), to evaluating organizations or activist groups (Vail, Arndt, Motyl, \& Pyszczynski, 2012), to aggression towards an outgroup member (McGregor et al., 1998), to intentions to use cannabis (Nagar \& Rabinovitz, 2015). Proponents of the theory claim these innovations represent a finetuning of the theory (Pyszczynski et al., 2015), whereas critics argue that TMT's predictions are too imprecise; heterogeneous, and often conflicting results can be framed as supportive evidence (Martin \& van den Bos, 2014).

Second, though the MS hypothesis frames death avoidance as central, manipulations that do not involve thoughts about death also elicit some comparable findings (e.g., Burris \& Rempel, 2004; Holbrook, Sousa, \& Hahn-Holbrook, 2011; Proulx \& Heine, 2006). Last, some have criticized the logic underlying TMT. In particular, its reliance on a 'death drive' as part of its 
explanatory framework has been called into question, alongside a purported misunderstanding of evolutionary principles (Kirkpatrick \& Navarrete, 2006).

The latter argument; that TMT resists explanation by other frameworks; is to some a source of skepticism, and to others, the very reason why it is so enticing. TMT challenges some key assumptions of other explanatory frameworks (see Baumeister \& Leary, 1995; Kirkpatrick \& Navarrete, 2006; Leary, Tambor, Terdal, \& Downs, 1995) and is therefore not derivative of any other model. This stand-alone model offers an encompassing explanatory framework for much of human behavior, and even some its critics concede that TMT represents an admirable effort to capture diverse phenomena, whereas most other frameworks carve out only a small corner of social psychology (e.g., Leary \& Schreindorfer, 1997).

We add a fourth reason to be skeptical of TMT: it is counterintuitive. This is part of the theory's appeal; people would not expect their behavior to derive from unconscious death thoughts, and would be surprised to learn of the mechanisms guiding their actions. Counterintuitive research in social psychology seems particularly popular, and as a result may attract more funding. One reason why counterintuitive findings may not replicate is that, being more prized, researchers are incentivized to resort to QRPs in order to obtain them. Another reason is that counterintuitive effects are more likely to correspond to small effect sizes, as one might expect that larger effects would be more observable in people's experiences, and smaller effects would more likely be dependent on $p$-hacking efforts in order to provide significant effects in studies with typical (small) sample sizes. This would result in an increase of the proportion of published findings that are false positives relative to true effects (see Ioannidis, 2005). 


\section{Replicability of Large Literatures}

The quality of the evidence in large literatures is often assessed with meta-analyses, and these can establish more precise effect size estimates, and inclusions of unpublished null findings have the potential to reveal biases in the published literature. But it is difficult to include the entire file drawer in a meta-analysis, and techniques developed for considering the size of an effect with respect to a potentially large file drawer (e.g., fail-safe N; Rosenthal, 1979) do not consider the influence of QRPs and inflated Type 1 error rates. These practices undermine the utility of such analyses and obscure any conclusions that can be drawn from the published literature (Simonsohn et al., 2014a). Because of these practices, the conclusions that are drawn from meta-analyses often overestimate the magnitude of true underlying effect sizes. This would also seem likely for the case of meta-analyses of the MS hypothesis.

A meta-analysis by Burke et al. (2010), using results from 277 individual studies, found that the average effect size for mortality salience studies is $r=.35$, corresponding to an effect of moderate size. Moreover, the authors also determined that the studies whose effects were largest were those that (1) used American samples, (2) used college student samples, (3) employed at least one delay task between the independent and dependent variables, and (4) tested the effects of death reminders on attitudes towards other people. Since it was published over a decade ago, this meta-analysis has been guiding TMT researchers in their decisions regarding study design and power planning. However, a limitation of the Burke et al. (2010) meta-analysis is that it relied upon the few techniques that were available at the time to confront publication bias (specifically, an analysis of the funnel plot). Moreover, a re-analysis revealed that experimenter effects accounted for a lot of variance (Yen and Cheng, 2013). Since 2010, more meta-statistical tools, and more bias correction techniques have been introduced (see Carter, Schönbrodt, 
Gervais, \& Hilgard, 2019). Here, we focus on a technique called the $p$-curve, introduced by Simonsohn et al. (2014a).

\section{$P$-curve}

The TMT literature has, like many other fields of psychology, adopted $p<.05$ as the "gold standard" to evaluate whether a result is noteworthy. This norm can result in publication bias because experiments that produce $p$-values below .05 are much more likely to be published than are those with $p$-values of .05 or above. Because the traditional meta-analytic approach depends on a representative sample of all studies for the effect size estimate, the low accessibility of null findings can lead to biased estimates of the population true effect. In situations when the true population effect is null, meta-analyses can sometimes still estimate a substantial effect by aggregating the false positives in the literature. And even when the true population effect does exist, the meta-analytic effect size estimate may still be inflated because of the same publication biases. These biases tend to be more prominent when the proportion of null findings is large, that is, when the power is low. These problems are then exacerbated by the numerous QRPs mentioned above.

Simonsohn et al. (2014a) created the $p$-curve to address the publication bias. The $p$-curve is, in essence, a histogram of all the $p$-values less than .05 in a body of literature. In the case where there is no population effect present, all $p$-values are equally likely (see a simulated distribution in Figure 1a). In contrast, when there is a true population effect, smaller $p$-values are more likely than larger $p$-values, forming a right skewed distribution (see Figure 1b) - the higher the power of the test, the more right skewed this distribution becomes. Therefore, Simonsohn et al. (2014a) suggest that when the histogram of the $p$-values in a literature shows the same height in each bin, it suggests a lack of evidential value in that literature. By the same principle, the 
presence of right skewness indicates the existence of a population effect, and the amount of the skewness measures the overall power of the studies. By testing it against a flat distribution, the $p$ curve can reveal whether $p$-values were most likely to be generated by a null effect. In contrast, by testing it against a distribution with a right skew corresponding to $33 \%$ power, the $p$-curve can reveal whether the studies in the literature have been conducted with sufficient power. Last, if the distribution contains a left skew, it can be taken as evidence that the literature may contain results that have been $p$-hacked. This is because the larger than expected cluster of $p$-values near the .05 cutoff suggests that values that were greater than .05 during the initial test were retested by using QRPs, which produced an up-tick in the distribution. The $p$-curve tool does not conduct inferential tests to diagnose significant left skew, but we present a $p$-hacked distribution for reference in Figure 1c.

\section{The Present Research}

We evaluate the evidential value in the overall literature of the MS hypothesis, the most prominent derivation of TMT. Using the $p$-curve, we assessed whether the published literature corresponds to a nonzero effect size, and whether the power predicted by the $p$-curve is consistent with effect size estimates from previous attempts to meta-analyze these effects. We adapted the code by Simonsohn $(2017)^{1}$ and created an interactive application that enables users to test evidential value in various subgroups of studies. We report the $p$-curve of the entire universe of studies, as well as of some select subgroups of studies. We pre-registered our methods for selecting manuscripts and $p$-values on $\mathrm{OSF}^{2}$.

\footnotetext{
1 Available via www.p-curve.com/Supplement/

${ }^{2}$ See https://osf.io/rdsm4/?view_only=9da1be8b55e7450e9c7ce82356d5b7b9
} 
As pre-registered, we examined five subgroups, two capturing features of the sample (undergraduate vs. non-undergraduate samples, and the location of the samples), two capturing features of the design (the type of dependent variable, and the inclusion of delays between the independent and dependent variables) and one capturing researchers' awareness of replicability (the year the study was conducted) ${ }^{3}$. Past theoretical arguments, empirical research, and metaanalytic evidence (see Burke et al., 2010) suggest that these subgroups may moderate the effect size. We review the influence of these pre-registered variables in the main text and in the $p$-curve application, and other potential moderators that may be of interest to researchers.

\section{Methods}

\section{Study Selection Criteria}

We followed the study search and selection procedures used in Burke et al. (2010), adapting it for the $p$-curve analysis where necessary. We conducted a database search on PsycINFO using terror management theory and/or mortality salience as key phrases. We also obtained papers from reference sections of prior reviews, and from a well-established TMT website (www.tmt.missouri.edu). This process yielded 1373 unique manuscripts, many of which contained multiple studies. We then applied the following screening rules: (1) manuscripts must be published in peer-reviewed journals, (2) manuscripts must be available in English, and (3) manuscripts must include novel empirical tests of the MS hypothesis (i.e., no review papers or meta-analyses). Moreover, we did not include studies published exclusively as part of a dissertation or thesis. On this basis, 527 manuscripts were initially screened from our analyses, and an additional 174 were removed after careful scrutiny, totalling 701 manuscripts (see Figure

\footnotetext{
${ }^{3}$ We also pre-registered that we would analyze subgroups of sample sizes; instead, we present estimated effect sizes based on each subset's average $\mathrm{N}$ and number of cells.
} 
2). The remaining manuscripts contained 1396 studies which were subjected to a stricter set of inclusion criteria.

\section{Inclusion Criteria}

A full list of excluded and included studies is available on $\mathrm{OSF}^{2}$.To be included in the $p$ curve analysis, studies needed to include: (1) a test of the MS hypothesis (this means that proximal defense and death-thought accessibility dependent measures do not qualify); (2) a true experimental manipulation of mortality salience with random assignment to condition, and (3) compatible with the $p$-curve method such that at least one statistical test, using statistics accepted by the tool, that was unambiguously attached to the primary hypothesis was $p<.05^{4}$. A further 578 individual studies were excluded for not meeting these criteria. After applying exclusions, 818 studies reporting complete statistical tests were suitable for the $p$-curve.

\section{$P$-value selection rules}

For each of the studies included, we selected one $p$-value associated with the focal hypothesis, which would be part of the $p$-curve. The fundamental considerations of the $p$-curve are that $p$-values correspond to tests of the focal hypothesis, and that they have been published under the criterion of $p<.05$. Due to the requirement that the $p$-value was selected under the $p<$ .05 publication pressure, additional rules apply when the focal hypothesis involves an interaction. When the interaction is attenuated; that is, the associated simple main effects are all in the same direction; the $p$-value associated with the interaction is selected (see Simonsohn et al., 2014a). This is because an attenuating interaction is always smaller than the largest simple main effect. Therefore, if a paper is deemed publishable because an attenuating interaction yields

\footnotetext{
${ }^{4}$ We also excluded studies whose primary hypothesis required a test of mediation, and no other result would have plausibly explained why the study was published, as there is no straightforward way to include a mediation model in the p-curve (which requires a single statistic).
} 
a $p<.05$, the accompanying simple main effect essentially contains a $p$-value that was selected based on a stricter criterion. In contrast, when the interaction is reversing; that is, the associated simple main effects are in different directions; the $p$-value associated with both simple main effects are selected. This is because the reversing interaction is always greater than the simple main effects. The full description of the selection rules can be found in the $p$-curve user guide (see Simonsohn, Nelson, \& Simmons, 2015).

We made small deviations from these guidelines, which are disclosed in our preregistration document ${ }^{2}$. Most notably, when the most appropriate test was not reported (e.g., reversing trends should be reported in the case of $2 \times 3$ interactions), we reported what the authors chose as the test of their key prediction; these were either interaction terms or main effects, depending on the nature of the hypothesis. Also, when reversing interactions were predicted, we reported two simple effects in accordance with recommendations from Simonsohn, Nelson, and Simmons (2015), unless the reversal was reported separately for the control and mortality salience conditions (not separated by levels of the interaction variable) in which case the simple effect in the control condition did not test the MS hypothesis. Here, we reported the interaction term. We made these deviations unless the context of the reporting suggested the publication criterion was not $p<.05$. Our goal was to be more inclusive of the entire literature, and to select as many $p$-values as possible that are associated with the main hypotheses of published papers. Last, when the paper reported tests for two or more non-independent hypotheses that were (1) equally prominent in the paper, (2) equally likely to lead to publication, and (3) equally related to the mortality salience hypothesis, we only included the first listed test in the results section. 
Finally, in accordance to the $p$-curve procedure by Simonsohn, Nelson, \& Simmons (2015), to account for any inaccurate and incorrect reporting, all $p$-values were recomputed using the test statistics (e.g., the $t$-statistics and the degree of freedom). Any study with recomputed $p$ values of .05 or above were excluded from the analyses.

\section{Overview of Analyses}

\section{Inferential tests for evidential value}

The shape of the $p$-curve provides a visual diagnostic for the evidential value of the body of literature. In order to test the shape of $p$-curve statistically to control for sampling error, Simonsohn, Simmons, and Nelson (2015) proposed two robust Stouffer's Z-tests, including a test for right skew and a test for flatness.

Test for right skew. When the $p$-curve appears to have at least some right skew visually, the right skew test can be used to statistically examine this conclusion. In this test, the null hypothesis is that the $p$-curve is flat, and thus a significant result indicates a right skewed distribution of the $p$-values (and hence, evidential value). For the selected $p$-value in each study, we compute the probability of obtaining a $p$-value at least as extreme as the one observed given a flat $p$-curve. This is essentially a $p$-value of that $p$-value, known as a $p p$-value. The $p p$-values are then aggregated using Stouffer's method, resulting in a $Z$-score. This $Z$-score then provides a single $p$ value for the right skew test. If $p p<.05$, the right skew test supports the conclusion that there is evidential value in the literature; otherwise, the right skew test does not provide such support.

Test for flatness. The null hypothesis of the flatness test is that the $p$-curve has a sufficient right skew; thus, a significant result indicates a flat distribution. Simonsohn et al. (2014a) define a sufficient right skew as one generated by studies with at least $33 \%$ power. The $p p$-values 
correspond to the probability of obtaining a $p$-value at least as extreme as the one observed given a distribution generated by at least $33 \%$ power. Though the principle is the same as in the right skew test, the interpretation is the opposite: Rejection of the null $(p p<.05)$ is evidence that the average power of the included study is below 33\%, while retention ( $\mathrm{pp} \geq .05)$ does not provide such evidence. Thus, significance in a flatness test indicates inadequate evidential value: Even if an effect exists, the extant body of literature is too insufficiently powered overall (significantly below 33\%) to provide satisfactory evidence for this effect, and direct replications are unlikely to succeed.

Binomial tests for right skew and flatness. In addition to these continuous tests, the pcurve also conducts binomial tests of right skew and flatness. Measuring right skew, the binomial test evaluates if the proportion of p-values above and below <.025 is significantly different from what would be expected if power was zero (because the distribution is flat, this value is $50 \%$ in both bins). Measuring flatness, the binomial test compares the distribution below .025 to one expected if power was $33 \%$ (approximately $71 \%$ ). The binomial test is intuitive, but is also insensitive to the distribution of p-values within each bin, and therefore is less diagnostic than the continuous tests (see Simonsohn et al., 2014a).

\section{Robust p-curve analysis}

A critical assumption of the $p$-curve is that the publication criterion is based on $p<.05$. Ulrich and Miller (2015) posited that the $p$-curve inferential tests may be biased by ambitious $p$ hacking: the act of selectively reporting based on criteria that are more stringent than $p<.05$ (e.g., reporting all results where $p<.04$, or simply always reporting only the lowest $p$-value). Simonsohn, Simmons, and Nelson (2015) noted that it is uncommon to report only the smallest $p$-value, and ambitious $p$-hacking to a criterion below .025 would be rare due to its difficulty. As 
a sensitivity analysis to account for moderately ambitious $p$-hacking such as $p<.03$ and $p<.04$, Simonsohn, Simmons, and Nelson (2015) implemented additional inferential tests based on the criterion of $p<.025$, known as the half $p$-curve analysis. These additional inference tests are similar to the right skew and flatness tests in the main analysis, except they are performed only on studies with $p$-values below .025 .

For a robust test for right skew, if the half $p$-curve test yields $p p<.05$, or if both the half $p$ curve and the full $p$-curve yield $p p<.10$, we consider there to be significant right skew, and take the result as evidence of the presence of evidential value. For a robust test for flatness, if the full $p$-curve yields $p p<.05$ or the half $p$-curve and binomial tests both yield $p p<.10$, we consider the curve to be significantly flatter than one that has $33 \%$ power, and take the result as evidence for inadequate evidential value. Although, we note that the lack of evidence for inadequate evidential value from a binary hypothesis test does not imply high evidential value: to aid us in our interpretations of the results, we also refer to the power estimates produced by the $p$-curve analysis.

\section{Power and effect size estimates}

A distinguishing feature of the $p$-curve is that it can be used to aggregate studies with different designs and analyses. A direct consequence, however, is that $p$-curve typically does not produce a straightforward average effect size estimate for the studies included ${ }^{5}$. In place of an effect size estimate, the $p$-curve produces an estimate of the average power of the studies by fitting the average power most likely to produce the observed $p$-curve (Simonsohn, Nelson, \& Simmons, 2014b).

\footnotetext{
${ }^{5}$ Further explorations of $p$-curve's advantages and limitations is left for the discussion.
} 
When a $p$-curve includes only one type of effect, Simonsohn et al. (2014b) also provide a blueprint for a procedure to estimate an effect size from a $p$-curve. However, since our $p$-curve encompasses a large variety of studies and effects, this approach is unavailable to us. In order to provide readers with a general sense of the effect sizes involved in a typical $p$-curved study, we use the estimated power to infer the corresponding effect sizes given some set of experimental conditions (see Table 3). To illustrate, when studies had two cells and a total of $N=115$ participants, we estimated the effect size from the $p$-curve power estimate (26\%) and $n$ per cell $(115 / 2)$ to be $d=0.25$. When studies reported a $2 \times 2$ attenuating interaction, requiring four cells, we estimated the effect size from power (26\%) and $n$ per cell $(115 / 4)$ to be $d=0.35$.

\section{Between-study moderators}

We point to some between-study moderators in our pre-registration ${ }^{2}$ (note we did not make specific predictions) and included others that may impact estimated power, which we identified based on theory and past meta-analytic evidence (see Burke et al., 2010; Pyszczynski et al., 2015). We list them in Table 1.

Affecting the interpretation of the statistic, we tracked if it corresponded to a main effect or interaction. We also report five features of the study design; the experimental manipulation, control condition, dependent variable type, number of delay tasks, and interaction type (if one was included). A minority of studies diverged from the "typical" experimental manipulation, called the Mortality Attitudes Perception Survey, in which participants had to write two paragraphs about what will happen to their physical bodies after they die, and the thoughts and feelings this exercise aroused in them. This prime was typically accompanied by a control version of the task instructing participants to imagine they were experiencing Dental Pain (see Rosenblatt et al., 1989). Therefore, the two subgroups of studies we identified were those that 
employed the "Mortality Attitudes Perception Survey" and "Other", and for the control condition, "Dental pain" and "Other" (see Table 1).

We also kept track of two features of the sample: the country of origin, and whether it was primarily college students vs. non-students. Last, to measure progress towards more replicable research, we included two subgroups; studies conducted before and after 2011 (we consider this a turning point in the field's consciousness of replicability; see Simmons et al., 2011; Wagenmakers, Wetzels, Borsboom, \& van der Maas, 2011).

\section{Accompanying Application and Disclosure Table}

To accompany the main $p$-curve, we have developed a Shiny application that will enable readers to explore a variety of subgroups of studies, and how power is estimated within each of these $^{6}$. The code of the application, the requisite data, as well as instructions on how to run it offline, are available on $\mathrm{OSF}^{2}$. We caution against multiple comparisons, which can inflate Type 1 error rates. We encourage users to pre-register their predictions and their theoretical or methodological reasoning (even privately) before using the accompanying app.

We also included a $p$-curve disclosure table on $\mathrm{OSF}^{2}$ (this is a formatted table with all available information needed to derive the $p$-curve).

\section{Results}

\section{Entire Universe of Studies}

The main analysis includes $K=818$ studies (see Figure 2 ) that satisfied all of the inclusion criteria. Because studies in the full sample involve idiosyncratic methods and diverse populations, the resulting heterogeneity of effect sizes will likely lead to a high false positive on

\footnotetext{
${ }^{6}$ Available at https://tmtpcurve.shinyapps.io/main/
} 
p-curves evidential value test (Carter et al., 2019). Consequently, we cannot make conclusive claims about the existence of evidential value in the literature based on the full sample.

Nevertheless, the main analysis will provide an overview of the health of the TMT literature, as well as a baseline for the subsequent subgroup analyses.

\section{Estimated power and inferred effect sizes}

Based on the shape of the $p$-curve, the average power of all 818 studies is estimated to be $26 \%, \mathrm{CI}_{90}=[22.2 \%, 30.4 \%]$. Simonsohn et al. (2014a) suggested using $33 \%$ power as the cutoff to decide whether the power of a body of literature is sufficient. Following this recommended cutoff, our main result suggests that tests of the MS hypothesis are generally underpowered in the literature (See Figure 3 and Table 2).

\section{Inferential tests}

The test for right skew determines that there is evidential value if the inferential test on the half curve (these are $p$-values smaller than .025) yields $p p<.05$, or both the full and half $p$-curves yield $p p<.10$. Using the half $p$-curve (all values below .025), we find significant right skew, $Z=$ $-14.18, p<.001^{7}$. Using the full $p$-curve, we also find significant right skew, $Z=-14.76, p<$ .001. Thus, we conclude there is evidential value in the overall literature, and there are nonzero effects underlying the studies on a whole.

The test for flatness evaluates whether there is adequate evidential value, which Simonsohn et al. (2014a) define as having an average estimated power of at least $33 \%$. The null of this test is that the $p$-curve has adequate evidential value, so a rejection means the $p$-curve is significantly flatter than one generated by $33 \%$ power. The test determines there is inadequate evidential value if the full curve yields $p p<.05$, or both the half curve and binomial test yield $p p$

\footnotetext{
${ }^{7}$ For convenience and consistency with the app, we refer to $p p$-values as $p$-values in our results.
} 
$<.10$. According to the full $p$-curve, the studies do not contain adequate evidential value: the curve is significantly flatter than one generated by $33 \%$ power, $Z=2.74, p=.003$. Using the half $p$-curve and binomial test, we do not find significant flatness $\left(Z_{\text {half }}=27.11\right.$, phalf $>.999, p_{\text {binomial }}<$ $.001)$, though we note the robust test requires only one of the conditions is satisfied; that the full $p$-curve is significantly flat, or the half $p$-curve and binomial tests are $p<.10^{8}$.

In sum, on the basis of these two inferential tests we can conclude that the entire universe of studies testing the MS hypothesis likely has evidential value, but the evidential value may be inadequate: should the effect exist, the modal study does not appear to be powerful enough to reliably detect and estimate the effect. The average $N$ in the universe of studies was 115 , which corresponds to 57.5 or fewer participants per cell; roughly enough to detect an effect size of $d=$ 0.50 with $80 \%$ power, or more realistically, an effect size of $d=0.25$ with $26 \%$ power.

\section{Moderating variables}

We evaluated the literature in subgroups to obtain sets of more homogeneous studies, following recommendations from Van Aert, Wicherts, and van Assen (2016). We identified variables that might influence power, from past meta-analyses (Burke et al., 2010) and theory (Pyszczynski et al., 2015). Below we report findings for those moderators we outline in our preregistration (though note we had no specific predictions about what shape the $p$-curves would take).

\section{Type of dependent variable}

Past meta-analytic evidence finds studies whose dependent variables are attitude measures are associated with the largest effect sizes (see Burke et al., 2010). Consistent with past research,

\footnotetext{
${ }^{8} \mathrm{We}$ also note that the test for flatness of the half $p$-curve is likely conservative; throughout, we find $p$-values corresponding to $>.999$.
} 
the highest powered subgroup was studies of people's attitudes and beliefs $(K=418)$, followed by studies evaluating affect and emotion $(K=65)$. For test of attitudes and beliefs, power was $31 \%, \mathrm{CI}_{90}=[25.0 \%, 37.1 \%]$ and there was significant right skew $\left(Z_{\text {full }}=-11.99, p_{\text {full }}<.001, Z_{\text {half }}\right.$ $\left.=-10.87, p_{\text {half }}<.001\right)$, but but was not significantly flatter than a curve generated by at least $33 \%$ power $\left(Z_{\text {full }}=-0.67, p_{\text {full }}=.253, Z_{\text {half }}=20.44, p_{\text {half }}>.999\right.$; the binomial test was significant at $p_{\text {binomial }}=.001$, though, recall this test makes use of less information than the continuous tests). Together, these findings suggest evidential value was present and also adequate. For affect as well, power was high at $\left.28 \%, \mathrm{CI}_{90}=[15.4 \%, 43.8 \%]\right)$, and there was significant right skew $\left(Z_{f u l l}\right.$ $\left.=-4.43, p_{\text {full }}<.001, Z_{\text {half }}=-3.97, p_{\text {half }}<.001\right)$, and not flatness $\left(Z_{\text {full }}=-0.55, p_{\text {full }}=.292, Z_{\text {half }}=\right.$ 7.38, phalf $>.999$, pbinomial $=.242)$.

The weakest effects were found for tests of neural and physiological outcome variables ( $\mathrm{K}$ $=23)$ and for tests of people's desires for objects or specific outcomes $(\mathrm{K}=88)$. For the neural and physiological subset, power was $12 \%, \mathrm{CI} 90=[5.0 \%, 34.3 \%]$. These studies passed the test for evidential value, based on a significant right skew for the half curve $\left(Z_{\text {full }}=-1.15, p_{\text {full }}=.124\right.$, $\left.Z_{\text {half }}=-1.69, p_{\text {half }}=.046\right)$, and had marginal flatness according to the full $p$-curve $\left(Z_{\text {full }}=-1.59\right.$, $\left.p_{f u l l}=.056\right)$. The half $p$-curve yields $p>.10\left(Z_{\text {half }}=3.77\right.$, $\left.p_{\text {half }}>.999\right)$, though the binomial test outcome is below the threshold (pbinomial $=.047$ ). On the basis of the half curve, the robust test retains the null of adequate evidential value, though we note that throughout our analyses the null is always retained in the half $p$-curve test for flatness (suggesting this test may be relatively insensitive). Given the marginal result associated with the full $p$-curve, and mixed evidence from the binomial test and half $p$-curve, we cautiously report there is adequate evidential value.

For the desire subset, power was $\left.18 \%, \mathrm{CI}_{90}=[9.6 \%, 29.7 \%]\right)$, and the inferential tests showed significant right skew $\left(Z_{\text {full }}=-3.53, p_{\text {full }}<.001, Z_{\text {half }}=-2.31, p_{\text {half }}=.011\right)$ as well as 
flatness $\left(Z_{\text {full }}=-2.09, p_{\text {full }}=.018, Z_{\text {half }}=7.58, p_{\text {half }}>.999\right.$, p $\left._{\text {binomial }}=.086\right)$ suggesting evidential value was inadequate.

\section{Number of Delays}

According to theory, studies with longer delays more effectively elicit distal responses; therefore, these studies should provide evidence for stronger worldview defense in the face of MS (Greenberg et al., 1994). The $p$-curve confirms this prediction: Studies with zero delays $(\mathrm{K}=124)$ produced the weakest effects; the $p$-curve corresponded to a power estimate of $23 \%, \mathrm{CI}_{90}=[14.3 \%, 34.2 \%]$. Though power was low, this subset was associated with significant right skew $\left(Z_{f u l l}=-5.17, p_{\text {full }}=.012, Z_{\text {half }}=-5.51, p_{\text {half }}<.001\right)$ and retains the null of at least $33 \%$ power $\left(Z_{\text {full }}=-1.52, p_{\text {full }}=.064, Z_{\text {half }}=10.77, p_{\text {half }}>.999\right.$, pbinomial $\left._{\text {b }}=.009\right)$ suggesting evidential value was present and adequate (though again, we note that the flatness test for the full $p$-curve yielded a marginal $p$-value, and for the binomial test yielded a significant $p$-value, so we are cautious in this interpretation). Studies with more than zero delays $(\mathrm{K}=635)$ produced stronger effects: power was estimated at $26 \%, \mathrm{CI} 90=[21.2 \%$, $30.6 \%$ ], closer to the modal study. Also consistent with the modal study, this subset had significant right skew $\left(Z_{\text {full }}=-12.84, p_{\text {full }}<.001, Z_{\text {half }}=-12.23, p_{\text {half }}<.001\right)$ but was significantly flat $\left(Z_{f u l l}=-2.57 p\right.$ full $=.005, Z_{\text {half }}=23.71$, phalf $>.999$, pbinomial $\left.<.001\right)$ suggesting evidential value, though present, was inadequate. The group with the strongest effects was those studies including two or more tasks separating the independent and dependent variables $(\mathrm{K}=151)$. These had greater power $\left(34 \%, \mathrm{CI}_{90}=[24.5 \%, 44.6 \%]\right)$, significant right skew $\left(Z_{\text {full }}\right.$ $\left.=-7.80, p_{\text {full }}<.001, Z_{\text {half }}=-7.02, p_{\text {half }}<.001\right)$, and not significant flatness $\left(Z_{\text {full }}=0.15, p_{\text {full }}=\right.$ $.562, Z_{\text {half }}=12.80$, phalf $>.999$, pbinomial $\left.=.166\right)$, suggesting adequate evidential value. 


\section{Country of origin}

Because Westerners are less interdependent (Markus \& Kitayama, 1991), and have fewer close connections that might provide sources of symbolic immortality (Park \& Pyszczynski, 2015; Pyszczynski et al., 2015), they are expected to have larger reactions to death thoughts in comparison with non-Westerners. Moreover, some non-Western religious traditions have been argued to provide better buffers against death thoughts (Park \& Choi, 2002; Park \& Pyszczynski, 2015), which would also predict larger worldview defense among Westerners. The meta-analysis by Burke et al. (2010) provided evidence in line with these predictions as the average $r$ was calculated to be .37 for studies conducted in the US, .30 for studies conducted in Europe or Israel, and .26 for studies conducted in Asia. However, the $p$-curve analysis was not in line with these findings: Studies conducted in the US were not more powerful than studies conducted in Europe and the Middle East, East Asia, or in Canada and Australia. In stark contrast to earlier meta-analytic evidence, the most powerful $p$-curve emerged in East Asia $(\mathrm{K}=55)$; power was estimated there as $58 \%, \mathrm{CI} 90=[42.4 \%, 71.6 \%]$ and the inferential tests suggested evidential value was present and adequate (Test for right skew: $Z_{\text {full }}=-7.50, p_{\text {full }}<.001, Z_{\text {half }}=-7.29, p_{\text {half }}<$ .001 ; Test for flatness: $Z_{\text {full }}=2.57, p_{\text {full }}=.995, Z_{\text {half }}=9.55$, phalf $>.999$, pbinomial $\left.=.439\right)$. In the US $(\mathrm{K}=455)$, on the other hand, power was estimated at $26 \%, \mathrm{CI}_{90}=[21.1 \%, 32.2 \%]$, and like the modal study, those belonging to this subset had significant right skew $\left(Z_{\text {full }}=-11.09, p_{\text {full }}<.001\right.$, $\left.Z_{\text {half }}=-10.15, p_{\text {half }}<.001\right)$ but also significant flatness $\left(Z_{\text {full }}=-1.98, p_{\text {full }}=.024, Z_{\text {half }}=20.04\right.$, pbinomial >.999) so evidential value was present but not adequate.

In Canada and Australia $(\mathrm{K}=62)$ power was estimated at $39 \%, \mathrm{CI}_{90}=[24.3 \%<55.4 \%]$. For these regions, the test for right skew was significant $\left(Z_{\text {full }}=-5.74, p_{\text {full }}<.001, Z_{\text {half }}=-4.18\right.$, $\left.p_{\text {half }}<.001\right)$ and the test for flatness was not $\left(Z_{\text {full }}=0.64, p_{\text {full }}=.739, Z_{\text {half }}=7.87, p_{\text {half }}>.999\right.$, 
p binomial $=.668)$ suggesting evidential value was present and adequate. In Europe and the Middle East $(\mathrm{K}=234)$, power was estimated as $17 \%, \mathrm{CI}_{90}=[11.6 \%, 24.1 \%]$, and as in the US, there was significant right skew $\left(Z_{\text {full }}=-5.52, p_{\text {full }}<.001, Z_{\text {half }}=-6.36, p_{\text {half }}<.001\right)$ as well as significant flatness $\left(Z_{\text {full }}=-3.53, p_{\text {full }}<.001, Z_{\text {half }}=13.41, p_{\text {half }}>.999, p_{\text {binomial }}<.001\right)$, suggesting evidential value was present but inadequate.

\section{Undergraduates vs. non-undergraduates}

In the past, some have argued that undergraduate samples should produce the strongest effects (Chopik, 2017; Cicirelli, 2001, 2002; Fortner \& Neimeyer, 1999), and the meta-analysis by Burke et al. (2010) provided evidence for this prediction. However, the $p$-curve does not confirm this prediction; among samples with undergraduates $(K=653)$ power was estimated as $24 \%, \mathrm{CI} 90=[20.0 \%, 28.9 \%]$. This $p$-curve corresponds to significant right skew $\left(Z_{f u l l}=-12.37\right.$, $\left.p_{\text {full }}<.001, Z_{\text {half }}=-12.30, p_{\text {half }}<.001\right)$ but also significant flatness $\left(Z_{\text {full }}=-3.12, p_{\text {full }} .001, Z_{\text {half }}=\right.$ 23.93, phalf $>.999$, pbinomial $<.001)$ suggesting evidential value was present but inadequate. In contrast, among non-undergraduate samples $(\mathrm{K}=156)$ power was estimated as $35 \%$, $\mathrm{CI} 90=$ $[25.5 \%, 44.9 \%]$ and the $p$-curve has significant right skew $\left(Z_{\text {full }}=-8.22, p_{\text {full }}<.001, Z_{\text {half }}=-7.13\right.$, $\left.p_{\text {half }}<.001\right)$ but not significant flatness $\left(Z_{\text {full }}=0.28, p_{\text {full }}=.609, Z_{\text {half }}=12.59, p_{\text {half }}>.999, p_{\text {binomial }}=\right.$ .104) suggesting evidential value was both present and adequate.

\section{Year of study}

One question we sought to answer with the p-curve is whether studies have improved in terms of their power since 2011. We chose 2011 as a cutoff because this is when the field developed a greater consciousness of the need for replicability (see Simmons et al., 2011; Wagenmakers et al., 2011). Further, a comprehensive meta-analysis was published in 2010 (see 
Burke et al., 2010) so this earlier subset may be informative of how the p-curve approach differs from traditional meta-analysis.

Among studies conducted before $2011(\mathrm{~K}=406)$ power was low at $24 \%, \mathrm{CI} 90=[18.1 \%$, 29.6\%]. The curve was significantly right skewed $\left(Z_{\text {full }}=-9.49, p_{\text {full }}<.001, Z_{\text {half }}=-9.23, p_{\text {half }}<\right.$ $.001)$ though there was also significant flatness $\left(Z_{\text {full }}=-2.66, p_{\text {full }}=.0039, Z_{\text {half }}=18.77, p_{\text {half }}>\right.$ .999 , p binomial $<.001$ ), suggesting evidential value was present but inadequate. Since 2011, things had improved somewhat; power for these later studies $(\mathrm{K}=412)$ was estimated at $29 \%, \mathrm{CI} 90=$ $[23.1 \%, 34.9 \%]$. There was significant right skew $\left(Z_{\text {full }}=-11.38, p_{\text {full }}<.001, Z_{\text {full }}=-10.82, p_{\text {half }}<\right.$ .001). In contrast to the subset of studies before 2011, studies since 2011 no longer yielded significant flatness under the continuous test $\left(Z_{\text {full }}=-1.23, p_{\text {full }}=.110, Z_{\text {half }}=19.57, p_{\text {half }}>.999\right.$, pbinomial $<.001$ ), suggesting evidential value was present and adequate. However, it is worth noting that the confidence intervals of the power estimates from the two year brackets overlap substantially, and the binomial test for the latter still indicated significant flatness.

For a more fine-grained look at how the average power of the studies changes over years, we computed the power estimates of the studies grouped into 6 bins by their year of publication: 1989-1994, 1995-1990, 2000-2004, 2005-2000, 2010-2014, and 2015+ (see Figure 4). There were relatively few studies during the early years, with $K=15$ during 1989-1994. The average power estimate for the studies in this period was relatively high initially, at $68 \%, \mathrm{CI}_{90}=[34.2 \%$, 87.7\%], with an average sample size of $\bar{N}=69$. During the subsequent year range, 1995-1999, the number of studies nearly tripled to $\mathrm{K}=44$. The average sample size remained similar at $\bar{N}=$ 73 , but the estimated power dropped to $37 \%, \mathrm{CI}_{90}=[18.8 \%, 56.0 \%]$. During $2000-2004$, the number of studies more than doubled from the previous year range, to $K=100$, but the power continued to decline, to $14 \%, \mathrm{CI}_{90}=[7.5 \%, 25.2 \%]$, despite an increase in average sample size to 
$\bar{N}=93$. In the subsequent years, the average sample size continued to climb, to $\bar{N}=101,112$,

161 , through the next three year ranges, as the power increased somewhat to $22 \%, \mathrm{CI} 90=[14.8 \%$, $30.0 \%], 24 \%, \mathrm{CI}_{90}=[17.4 \%, 31.4 \%]$, and finally to $35 \%, \mathrm{CI} 90=[26.7 \%, 44.2 \%]$ for all studies since 2015. The pattern of increasing power after the field began to take QRPs seriously bodes well for TMT.

\section{Additional exploratory tests}

\section{Main effect or interaction}

We coded studies for whether the results reported as the primary hypothesis correspond to main effects or interactions. We determined that studies that predicted main effects $(K=191)$ had substantial power $\left(48 \%, \mathrm{CI}_{90}=[38.2 \%, 56.2 \%]\right)$. The inferential tests suggest there was significant right skew $\left(Z_{\text {full }}=-11.46, p_{\text {full }}<.001, Z_{\text {half }}=-12.09, p_{\text {half }}<.001\right)$ but not flatness $\left(Z_{\text {full }}=\right.$ 2.53, $p_{\text {full }}=.994, Z_{\text {half }}=16.13$, phalf $>.999$, pinomial $\left.=.0249\right)$ so evidential value was present and adequate. In contrast, the studies that predicted interactions $(K=608)$ had considerably less power $\left(19 \%, \mathrm{CI}_{90}=[15.4 \%, 23.6 \%]\right)$, and like the modal study, though the $p$-curve has significant right skew $\left(Z_{\text {full }}=-9.93, p_{\text {full }}<.001, Z_{\text {half }}=-8.69, p_{\text {half }}<.001\right)$ it also had significant flatness $\left(Z_{\text {full }}=-4.87\right.$, pull $<.001, Z_{\text {half }}=21.26$, phalf $>.999$, pbinomial $\left.<.001\right)$ so evidential value was inadequate. These results suggest that studies that are testing for interactions do not have large enough samples per condition. The differences in power between tests of main effects and tests of interactions mirrors the principle that interactions require much larger samples, and inattention to this requirement causes studies to be underpowered (Aiken, West, \& Reno, 1991; Simonsohn, 2014). 


\section{Experimental manipulation and controls}

The most common manipulation of mortality salience is the Mortality Attitudes Personality Survey (MAPS; Rosenblatt et al., 1989), which involves two short-answer questions prompting participants to write about what will happen to them as they physically die, and then to jot down the emotions that these ideas arouse in them. Likewise, the most common control condition that is used is for participants to encounter a parallel prompt about dental pain.

Studies that used these common manipulations did not appear to be more powerful than the average. The subset $(\mathrm{K}=246)$ that used both the modal manipulation (MAPS) and dental control had power estimated at only $18 \% \mathrm{CI}_{90}=[12.7 \%, 25.4 \%]$. Though there was significant right skew $\left(Z_{\text {full }}=-6.06, p_{\text {full }}<.001, Z_{\text {half }}=-4.64, p_{\text {half }}<.001\right)$ there was also significant flatness $\left(Z_{\text {full }}=\right.$ $-3.30, p_{\text {full }}<.001, Z_{\text {half }}=13.12$, phalf $>.999$, p $\left._{\text {binomial }}<.001\right)$ suggesting evidential value was present but inadequate. Studies employing neither of these experimental or control conditions $(K=214)$ were associated with higher power $\left(27 \%, \mathrm{CI}_{90}=[19.6 \%, 35.7 \%]\right)$, and unlike studies employing a more typical design, they had both significant right skew $\left(Z_{\text {full }}=-7.79, p_{\text {full }}<.001, Z_{\text {half }}=-8.00\right.$, $\left.p_{\text {half }}<.001\right)$ and non-significant flatness $\left(Z_{\text {full }}=-1.19, p_{\text {full }}=.118, Z_{\text {half }}=14.23, p_{\text {half }}>.999, p_{\text {binomial }}\right.$ $=.003)$ suggesting evidential value was adequate.

\section{Interaction Type}

For studies that included interactions, we also evaluated subsets whose interactions were individual difference measures (traits, attitudes), gender, or features of the design (changing the manipulation, or the attitude target). We found the lowest power estimate in the subset whose moderator was an attitude measure $(\mathrm{K}=86): 12 \%, \mathrm{CI}_{90}=[6.2 \%, 21.5 \%]$. The inferential tests showed there was not significant right skew according to the half curve $\left(Z_{\text {full }}=-2.30, p_{\text {full }}=.011\right.$, $\left.Z_{\text {half }}=-0.63, p_{\text {half }}=.266\right)$ and there was also significant flatness $\left(Z_{\text {full }}=-3.12, p_{\text {full }}<.001, Z_{\text {half }}=\right.$ 
6.49$, phalf $>.999$, p binomial $=.008)$ so evidential value was neither present nor adequate. We speculate that some studies in this category may have employed QRPS; specifically, adding a post hoc moderator to yield $p<.05$; explaining the flat $p$-curve. For example, if researchers measured people's attitudes and worldviews during the study, but did not plan to analyze these, they may have added them as moderators after finding nonsignificant evidence for their primary predictions. On the other hand, the highest power was estimated in the subset of studies whose moderating variable was gender $(\mathrm{K}=57)$. Power was $31 \%, \mathrm{CI}_{90}=[15.7 \%, 48.1 \%]$, and the inferential tests showed significant right skew $\left(Z_{\text {full }}=-4.44, p_{\text {full }}<.001, Z_{\text {half }}=-5.22, p_{\text {half }}<.001\right)$ but not flatness $\left(Z_{\text {full }}=-0.30, p_{\text {full }}=.381, Z_{\text {half }}=8.17\right.$, phalf $>.999$, pbinomial $\left.=.024\right)$.

\section{Discussion}

The current $p$-curve analysis incorporated 818 studies to evaluate the evidential value in the literature. We believe it is the largest set of studies to date that have been analyzed with a $p$ curve. The conclusions that we draw from these analyses are both cautionary and encouraging. Overall, the $p$-curve analyses showed that there is evidential value in the MS hypothesis in both the entire universe of studies and within each of the several subgroups that were tested. This suggests that there is indeed a reliable effect in the literature testing the MS hypothesis. On the other hand, these analyses also showed that across the entire universe of studies there is not inadequate evidential value. Moreover, there was not inadequate evidential value in many (but not all) of the subgroup analyses. This indicates that the typical study that tested the MS hypothesis was generally underpowered, and that many of these studies likely relied on some QRPs.

In the spirit of open and transparent science, we released a Shiny application to accompany this paper ${ }^{6}$. This application loads the disclosure table (also available at $\mathrm{OSF}^{2}$ ) and performs $p$ - 
curve analyses on a user-defined subset of data based on any combination of moderators. Our intention is to provide an open, interactive implementation of the multiverse analyses (Steegen, Tuerlinckx, Gelman, \& Vanpaemel, 2016), to allow readers to critique our conclusions as well as to draw their own. Our vision for this application is that it can help researchers to evaluate the evidence for their pre-registered predictors, though we acknowledge it may facilitate HARKing if the application is used in an exploratory way. We strongly encourage people to use the application with caution, and avoid framing findings from exploratory investigations as confirmation of their a priori predictions. We hope that responsible use of this application will enable the research community to assess whether any particular conclusion is sensitive to arbitrary researcher decisions.

Our own pre-registered analyses investigated the influence of five moderating variables: 1) the type of dependent variable measured, 2) the number of delays between the manipulation and presentation of the dependent measure, 3) reliance on undergraduate vs. non-undergraduate samples, 4) the cultural context (country of origin) of the sample, and 5) the year in which the study was conducted. Our findings regarding the dependent variable type conformed to past meta-analytic evidence (Burke et al., 2010): Studies measuring attitudes and beliefs yielded the largest estimated power. Further, our findings regarding delays align with past meta-analytic evidence: Studies with zero delays are associated with less power overall, and studies with more than one delay were associated with higher levels of power. This converges with the pattern identified by Burke et al. (2010), where studies with zero delays had an average $r=.30$, and this value increased alongside greater delays to the point that studies with three delay tasks had an average $r=.47$. 
Our analyses of the sample-level moderators did not converge with that found in the metaanalysis by Burke et al. (2010). With respect to the difference between studies that relied on undergraduate vs. non-undergraduate samples, our $p$-curve analysis revealed lower power in the studies that relied on undergraduates than in the studies that recruited non-undergraduates. In contrast, Burke et al. (2010) found that the average effect size for undergraduates $(r=.36)$ was larger than that from studies involving non-undergraduates $(r=.25)$. With respect to the location of the studies, our $p$-curve analysis revealed that East Asian samples had greater power than did studies conducted elsewhere. In contrast, Burke et al. (2010) found that effect sizes were largest in the US and smallest in East Asia.

The differences between the results of the $p$-curve analyses and the Burke et al. (2010) meta-analysis for the different subgroups are difficult to interpret. After all, the analyses are evaluating the studies in quite different terms: the $p$-curve assesses the average power of a study, whereas a meta-analysis is estimating the average effect size. In general, we would expect that power and effect size should rise together in tandem, however, they may depart from each other if researchers have engaged in QRPs and are halting data collection once they have reached the threshold of $p<.05$.

However, it is encouraging that the results of both the present $p$-curve analysis and the meta-analysis of Burke et al. (2010) converge with the theoretical predictions that the MS hypothesis is best tested with a dependent variable corresponding to beliefs or attitudes, and when there is a substantial delay (i.e., greater than 2 delay tasks) between the independent and dependent variables (see Greenberg et al., 1994). This suggests that researchers would be recommended to focus on attitudes (and / or emotions), and to include more than one delay in future tests of the MS hypothesis. 
The average power of the studies over the years yielded two striking observations. First, as TMT gained popularity during 1989-2004, the estimated power rapidly declined, even though the average sample size slightly increased. It may be the case that, as researchers expanded the original design and explored the MS hypothesis in new contexts, the average effect of these new studies was much weaker compared to any potential effect under the strict protocol of the original study. Second, there has been a trend of steady improvement during the past 20 years. This trend provides optimism in drawing conclusions about the MS hypothesis. Though, we also caution that even the subset of all studies since 2015 was associated with relatively low power and evidential value. Efforts to address past failures to replicate have no doubt improved the quality of research; still, we advocate for an even larger push for practices that enhance replicability. The most striking among our exploratory findings was that main effects were so much more highly powered than were studies that investigated interactions, given that tests of main effects should be more sensitive than tests of interactions. These findings also point to a more encouraging assessment of the TMT literature, as they suggest that when TMT studies are designed in the most justifiable and sensitive ways, they are especially likely to provide evidential support for the theory.

\section{Comparing $\boldsymbol{P}$-curve and Traditional Meta-Analysis}

$P$-curve analyses and traditional meta-analyses are different in a number of respects. A key distinction between the two lies in their respective selection rules, which dictate how researchers select which studies to include. First, $p$-curve analyses can only be applied to $p$-values which are less than .05 , and thus cannot be applied to studies that have failed to reject the null hypothesis. In contrast, meta-analyses can include any $p$-values, regardless of whether they do or do not 
reject the null hypothesis. Hence, these two analyses will cover somewhat different collections of studies.

Second, traditional meta-analytic techniques analyze effects that were calculated with the same statistical designs and analyses. For example, a traditional meta-analysis can be conducted on a set of simple main effects, or interactions, but not both. In contrast, the effects selected for p-curve must be from each study's respective focal hypothesis (Simonsohn, Simmons, \& Nelson, 2015), regardless of whether the effect is a main effect or an interaction. This requirement follows from the $p$-curve's critical assumption that the publication bias works on a threshold of $p$ $<.05$, as $p$-values associated with non-focal hypotheses may not follow that assumed selection threshold. A further implication of $p$-curves $p<.05$ assumption is that for studies with attenuating interactions, that is, an interaction that is smaller than the largest simple main effect, the main effect cannot be used (see Simonsohn, Simmons, \& Nelson, 2015). The differences in the selection rule of $p$-curve and traditional meta-analyses, in terms of which effects to include, can lead to various advantages and weaknesses in each approach.

\section{Advantages of p-curve}

In the current study, we aimed to perform a comprehensive assessment of the TMT literature as a whole, and provide a tool to perform the same analysis on subgroups of studies defined by study characteristics. $P$-curve offered advantages that are more closely aligned with the goal of the current study. By analyzing the focal hypothesis, $p$-curve provides a more direct assessment of the evidential values in the main conclusions of the studies. In the studies we examined, the main conclusions of a majority of the studies (608 out of 818 ) are based on significant interactions: A meta-analysis of the simple main effects in these studies would not make for a good indicator whether the conclusions of these papers are supported by tests with 
evidential value. Further, the $p$-curve provides a more inclusive synthesis of the literature. Due to its selection rule, the $p$-curve is able to incorporate effects of different kinds, allowing the inclusion of more findings of theoretical interest in the analysis. This broader inclusivity also provides us with the necessary sample sizes to examine various subsets of studies based on theoretically meaningful criteria such as the driving theory, study stimuli, and demographics of participants.

\section{Limitations of p-curve}

The $p$-curve's selection rules also impose some limitations on the current investigation. The primary limitation is that, since our analyses combined effects obtained from various designs and analyses, testing different theoretical effects, they do not produce straightforward effect size estimates. Consequently, our study does not offer concrete guidelines for the power planning of future TMT studies. To provide some reference in terms of effect size estimates, we provide the power estimates, but we caution against interpreting these effect sizes especially when the corresponding $p$-curve does not pass the flatness test-we cannot rule out the likely scenario that these $p$-curves were influenced by selective reporting.

It should also be noted that when a $p$-curve includes studies with heterogeneous random effects, it more likely yields false positives pointing to a nonzero average effect size, when it is in fact zero (Carter et al., 2019). The false positive error rate increases as a larger number of effects are incorporated into the study. Because the main $p$-curve presented in this paper includes 818 studies on different aspects of the MS effect in diverse populations from all over the world, the main conclusion that there is an overall effect in the literature should be viewed in the context of the subgroup analyses, where the number of studies is smaller and the effects are more likely to be homogeneous. 
Since the publication of the original $p$-curve papers, alternative approaches for the correction of publication bias in meta-analysis have been garnering more attention, such as PETPEESE (Stanley \& Doucouliagos, 2014) and the three-parameter selection model (Iyengar \& Greenhouse, 1988; McShane, Böckenholt, \& Hansen, 2016). Because the employment of these traditional meta-analytic strategies requires a different set of selection criteria, we were unable to additionally perform these analyses on the studies and effects we have selected for the $p$-curve. Though no approach is without limitations, employing multiple strategies - some relying on the logic of meta-analysis, and others relying on $p$-curve or other alternative meta-statistical methods (see Bartoš \& Schimmack, 2021; Brunner \& Schimmack, 2020; Van Assen, Van Aert, \& Wicherts, 2015) - should provide a better overview of the health of the literature.

\section{Comment on previous failures to replicate MS effects}

The $p$-curve points to the presence of nonzero effects, that are larger when investigated with the most appropriate methods (i.e., two or more delays, studies designed to test for main effects, and studies conducted after researchers became more aware of the problems of QRPs), but has been frequently pursued in severely underpowered studies. Our findings are therefore more optimistic about the MS hypothesis than failed replications pointing to a null effect (see Klein et al., 2019; Rodríguez-Ferreiro et al., 2019; Sætrevik \& Sjåstad, 2019; Schindler et al., 2021). The weaknesses of the $p$-curve discussed above suggest we cannot discount the possibility that these studies amount to a null effect; though, we note that some recent pre-registered replications have been successful (e.g., Hayes \& Schimel, 2018) which also aligns with our findings. 


\section{Recommendations}

Together, these findings caution that researchers should not plan their studies to align with standards in place since before the field developed a greater consciousness of replication (see John et al., 2012; Munafò et al., 2017; Simmons, Nelson, \& Simonsohn, 2011). They might also align with critiques that TMT produces imprecise predictions, and sometimes contradictory outcomes can be framed as evidence supporting its logic (Martin \& van den Bos, 2014). If these moderating variables, ostensibly supported by theory, do not produce consistent outcomes, this could point to an inherent weakness in TMT. At the very least, we warn that theory refinement should not follow the discovery of new evidence, unless that evidence emerges consistently after pre-registered replications.

Though the $p$-curve analysis is optimistic of an effect of mortality salience, the effect size estimates have been grossly overestimated, likely as an outcome of publication bias and QRPs (Ioannidis, 2005; John et al., 2012; Kerr, 1998; Simmons et al., 2011; Simonsohn et al., 2014a). Therefore, we align ourselves with critics advocating for a more open science, and offer specific recommendations to TMT researchers.

\section{Power planning}

Regardless of the precedent set by previous studies, researchers should plan for small effects. Our $p$-curve suggests that in most cases, planning power on the basis of effect size estimates from past studies will likely yield null findings. Illustrating this phenomenon, a recent failed (pre-registered) replication set their sample size to be $\mathrm{N}=101$; larger than the typical MS experiment, but not large enough to detect a small effect $(d=0.30$ with only $31.7 \%$ power; Sætrevik \& Sjåstad, 2019). Notably, their second study included a much larger sample $(\mathrm{N}=784$, large enough to detect $d=0.30$ with $98.7 \%$ power) and still turned up null findings. Researchers 
should plan for a much smaller effect than what they may assume from past research, and eschew conventions set by their predecessors.

\section{Consistent and cumulative moderators}

A debate among critics and TMT researchers has been whether TMT evades falsification by integrating sometimes contradictory findings into the theory (Martin \& van den Bos, 2014), or the MS hypothesis has been refined and fine-tuned as hidden moderators have been discovered (Pyszczynski et al., 2015). Of the 818 studies we analyzed, 608 predicted the effect would be moderated by a second independent predictor. Supporting critics, these moderators were heterogeneous; people's chance of defending their worldview sometimes depended upon their gender; sometimes their national identity; sometimes cognitive capacities; and sometimes tweaks to the manipulation or dependent measure explained why an effect emerged in some but not all conditions. These measures and tweaks were inconsistent across studies, so they largely did not amount to theory refinement. Further, our analyses revealed that tests of main effects were more powerful than tests of these interactions. It seems quite likely that in many of these studies, researchers relied upon hidden moderators to $p$-hack their way to a significant effect, and the predictions of TMT are imprecise enough to invite researchers to speculate on how their findings align with theory.

\section{Theory refinement accompanied by strong rationale and (pre-registered predictions)}

The search for hidden moderators may lead researchers astray (though we agree with proponents of TMT that refining theory and its predictions are worthy pursuits; see Pyszczynski et al., 2015). We recommend two practices that can guide researchers towards more robust refinements. First, researchers should pre-register their predictions of moderators to avoid the pitfalls of HARKing. Second, researchers should include moderators that are culturally 
appropriate; if samples have specific or idiosyncratic worldviews, researchers must be sensitive to these idiosyncrasies, and justify the relevance of measures to their sample in advance of the study. A critique of large-scale replications like Many Labs 4 (see Klein et al., 2019) is that they may be insensitive to sample characteristics. Given that worldviews are cultural (or even individual) TMT researchers should make theoretically-sound arguments about how their samples' characteristics can impact results, accompanied by pre-registered predictions, in advance of a study.

\section{Comment on theory importance}

Some may see our findings as challenging the importance of TMT; if effects are as weak as the $p$-curve implies, and especially if the effects only emerge from strict adherence to the protocol (see Chatard et al., 2020), then perhaps death thoughts might explain less about human behavior than previously believed. On the other hand, we note that there is a key difference between the ways that thoughts affect people's behaviors and the ability of researchers to detect those effects in the context of a lab-based or online study. We can, of course, only evaluate the evidence that has emerged as a result of these research efforts. Nonetheless, the overall weak effects found in studies that test the MS hypothesis would seem to call into question early articulations of TMT as an organizing principle that explains the origins of such foundational human characteristics as culture and self-esteem (see Greenberg et al., 1986; Solomon et al., 1991). If effect sizes can serve as a guide for interpreting the field's conclusions about which psychological phenomena are most foundational, the evidence is mounting against these early claims that many behaviors emerged as a product of efforts to suppress death thoughts.

It is worth noting that our $p$-curve analysis focuses on the MS hypothesis, derived from TMT, but is not itself a test of the overarching theory. For this reason, many phenomena may yet 
have an existential origin that are not adequately tested through the MS hypothesis. Other predictions of TMT; for example, that worldview-violating events disrupt the buffer that holds thoughts of death at bay (as in the Death Thought Accessibility literature; Florian, Mikulincer, \& Hirschberger, 2002; Schimel, Hayes, Williams, \& Jahrig, 2007) may be informative, and we encourage future researchers to use meta-statistical tools like the $p$-curve to evaluate the merits of that literature. It is outside the scope of our analysis to comment on the merits of TMT as a whole, or compare it to alternative accounts of MS effects (see Heine, Proulx, \& Vohs, 2006; Jonas et al., 2014; Van Den Bos, 2001). In general we advise that those interested in fine tuning, or challenging the theory, employ more diverse methods that can contribute to a more holistic account of existential psychology.

\section{Conclusion}

Our $p$-curve analysis suggests that effect size estimates of the MS hypothesis have been overestimated by other meta-statistical techniques (e.g., Burke et al., 2010), though it points to a clear nonzero effect of mortality salience on worldview defense. Moreover, we found stronger evidence in support of the MS hypothesis in the most appropriately designed studies (i.e., studies measuring attitudes, studies employing theoretically-derived moderators of needing sufficient delays between the independent and dependent variable, studies employing more sensitive designs of main effects over ones that tested for interactions, and studies conducted since the field developed more awareness about the harmful effects of QRPs) is especially encouraging for evaluating TMT. While the recent publication of several failed replications of TMT studies may have led some to expect that our $p$-curve analysis of the TMT literature would result in an autopsy of the field, we have instead detected a clear pulse. The overall state of the field may not 
be as healthy as its most enthusiastic champions have suggested, but it is alive, and we hope future researchers design their studies with the results of this $p$-curve analysis in mind.

Though our analysis finds that the theory lives, we urge that the use of QRPs and the field's publication bias must be laid to rest. We find wisdom in the concept that some beliefs are hard to bury; challenging worldviews has negative cascading effects, such as threatening one's self-esteem, and eliciting dogmatic attachment to one's prejudices. Psychology researchers might have deep attachments to foundational studies, or to conventions that simplify the process of sample selection, power planning, and data analysis. It is difficult, and even existentially threatening, to overcome these attachments. We hope that our $p$-curve and the accompanying application ease the transition into a more open and accountable psychology. 


\section{References}

Aiken, L. S., West, S. G., \& Reno, R. R. (1991). Multiple regression: Testing and interpreting interactions. Sage.

Bartoš, F., \& Schimmack, U. (2021). Z-curve.2.0: Estimating the replication and discovery rates. Meta-Psychology, in press.

Baumeister, R. F., \& Leary, M. R. (1995). The need to belong: desire for interpersonal attachments as a fundamental human motivation. Psychological bulletin, 117(3), 497.

Becker, E. (1973). The denial of death. New York: Free Press.

Brunner, J. \& Schimmack, U. (2020). Estimating population mean power under conditions of heterogeneity and selection for significance. Meta-Psychology, 4, https://doi.org/10.15626/MP.2018.874

Burke, B. L., Kosloff, S., \& Landau, M. J. (2013). Death goes to the polls: A meta-analysis of mortality salience effects on political attitudes. Political Psychology, 34(2), 183-200. doi: 10.1111/pops. 12005

Burke, B. L., Martens, A., \& Faucher, E. H. (2010). Two decades of terror management theory: A meta-analysis of mortality salience research. Personality and Social Psychology Review, 14(2), 155-195. doi: 10.1177/1088868309352321

Burris, C. T., \& Rempel, J. K. (2004). "It's the end of the world as we know it": threat and the spatial-symbolic self. Journal of personality and social psychology, 86(1), 19.

Carter, E. C., Schönbrodt, F. D., Gervais, W. M., \& Hilgard, J. (2019). Correcting for bias in psychology: A comparison of meta-analytic methods. Advances in Methods and Practices in Psychological Science, 2(2), 115-144. doi: 10.1177/2515245919847196 
Chatard, A., Hirschberger, G., \& Pyszczynski, T. (2020). A Word of Caution about Many Labs 4: If You Fail to Follow Your Preregistered Plan, You May Fail to Find a Real Effect. doi: 10.31234/osf.io/ejubn

Chopik, W. J. (2017). Death across the lifespan: Age differences in death-related thoughts and anxiety. Death studies, 41(2), 69-77.

Cicirelli, V. G. (2001). Personal meanings of death in older adults and young adults in relation to their fears of death. Death studies, 25(8), 663-683.

Cicirelli, V. G. (2002). Fear of death in older adults: Predictions from terror management theory. The Journals of Gerontology Series B: Psychological Sciences and Social Sciences, 57(4), P358-P366.

Collaboration, O. S., et al. (2015). Estimating the reproducibility of psychological science. Science, 349(6251).

Dunn, L., White, K., \& Dahl, D. W. (2020). A little piece of me: When mortality reminders lead to giving to others. Journal of Consumer Research, 47(3), 431-453.

Dunne, S., Gallagher, P., \& Matthews, A. (2015). Existential threat or dissociative response? Examining defensive avoidance of point-of-care testing devices through a Terror Management Theory framework. Death Studies, 39(1), 30-38. doi: $10.1080 / 07481187.2014 .885469$

Fergus, T. A., \& Valentiner, D. P. (2012). Terror management theory and scrupulosity: An experimental investigation. Journal of Obsessive-Compulsive and Related Disorders, 1(2), 104-111. doi: 10.1016/j.jocrd.2012.01.003 
Florian, V., Mikulincer, M., \& Hirschberger, G. (2002). The anxiety-buffering function of close relationships: Evidence that relationship commitment acts as a terror management mechanism. Journal of Personality and Social Psychology, 82(4), 527-542. doi:

$10.1037 / 0022-3514.82 .4 .527$

Fortner, V., \& Neimeyer, R. B. (1999). Death anxiety in older adults: A quantitative review. Death studies, 23(5), 387-411.

Giofrè, D., Cumming, G., Fresc, L., Boedker, I., \& Tressoldi, P. (2017). The influence of journal submission guidelines on authors' reporting of statistics and use of open research practices. PloS one, 12(4), e0175583.

Greenberg, J., \& Helm, P. J. (2019). Terror Management Theory and its implications for older adults. Encyclopedia of Gerontology and Population Aging (Lifton 1979), 1-5. doi: 10.1007/978-3-319-69892-2_115-1

Greenberg, J., Pyszczynski, T., \& Solomon, S. (1986). The causes and consequences of a need for self-esteem: A terror management theory. In (p. 189-212). New York, NY: Springer New York.

Greenberg, J., Pyszczynski, T., Solomon, S., Simon, L., \& Breus, M. (1994). Role of consciousness and accessibility of death-related thoughts in mortality salience effects. Journal of Personality and Social Psychology, 67(4), 627-637. Doi: 10.1037/00223514.67.4.627

Hayes, J., \& Schimel, J. (2018). Unintended effects of measuring implicit processes: The case of death-thought accessibility in mortality salience studies. Journal of Experimental Social Psychology, 74(November 2017), 257-269. doi: 10.1016/j.jesp.2017.10.005 
Heine, S. J., Harihara, M., \& Niiya, Y. (2002). Terror Management in Japan. Asian Journal of Social Psychology, 5(3), 187-196.

Heine, S. J., Lehman, D. R., Markus, H. R., \& Kitayama, S. (1999). Is there a universal need for positive self-regard? Psychological review, 106(4), 766.

Heine, S. J., Proulx, T., \& Vohs, K. D. (2006). The meaning maintenance model: On the coherence of social motivations. Personality and Social Psychology Review, 10(2), 88110. doi: 10.1207/s15327957pspr1002_1

Hofstede, G. (2001). Culture's consequences: Comparing values, behaviors, institutions and organizations across nations. Sage publications.

Holbrook, C., Sousa, P., \& Hahn-Holbrook, J. (2011). Unconscious vigilance: Worldview defense without adaptations for terror, coalition, or uncertainty management. Journal of personality and social psychology,101(3), 451.

Ioannidis, J. P. A. (2005). Why most published research findings are false. PLOS Medicine, 2(8), null. doi: 10.1371/journal.pmed.0020124

Iyengar, S., \& Greenhouse, J. B. (1988). Selection models and the file drawer problem. Statistical Science, 109-117.

John, L. K., Loewenstein, G., \& Prelec, D. (2012). Measuring the prevalence of questionable research practices with incentives for truth telling. Psychological science, 23(5), 524532.

Jonas, E., McGregor, I., Klackl, J., Agroskin, D., Fritsche, I., Holbrook, C., ... Quirin, M. (2014). Threat and defense: From anxiety to approach. In Advances in experimental social psychology (Vol. 49, pp. 219-286). Elsevier. 
Jonas, E., Schimel, J., Greenberg, J., \& Pyszczynski, T. (2002). The scrooge effect: Evidence that mortality salience increases prosocial attitudes and behavior. Personality and Social Psychology Bulletin, 28(10), 1342-1353. doi: 10.1177/014616702236834

Kerr, N. L. (1998). Harking: Hypothesizing after the results are known. Personality and Social Psychology Review, 2(3), 196-217. (PMID: 15647155) doi: $10.1207 / \mathrm{s} 15327957 \mathrm{pspr} 0203 \backslash 4$

Kirkpatrick, L. A., \& Navarrete, C. D. (2006). Reports of My Death Anxiety Have Been Greatly Exaggerated: A Critique of Terror Management Theory from an Evolutionary Perspective. Psychological Inquiry, 17(4), 288-298. doi: 10.1080/10478400701366969

Klein, R. A., Cook, C. L., Ebersole, C. R., Vitiello, C. A., Nosek, B. A., Chartier, C. R., ... et al. (2019, Dec). Many labs 4: Failure to replicate mortality salience effect with and without original author involvement. PsyArXiv. doi: 10.31234/osf.io/vef2c

Leary, M. R., \& Schreindorfer, L. S. (1997). Unresolved issues with terror management theory. Psychological Inquiry, 8(1), 26-29.

Leary, M. R., Tambor, E. S., Terdal, S. K., \& Downs, D. L. (1995). Self-esteem as an interpersonal monitor: The sociometer hypothesis. Journal of personality and social psychology, 68(3), 518.

Lipset, S. M. (1996). American exceptionalism: A double-edged sword. WW Norton \& Company.

Markus, H. R., \& Kitayama, S. (1991). Culture and the self: Implications for cognition, emotion, and motivation. Psychological review, 98(2), 224. 
Martens, A., Burke, B. L., Schimel, J., \& Faucher, E. H. (2011). Same but different: Metaanalytically examining the uniqueness of mortality salience effects. European Journal of Social Psychology, 41(1), 6-10. doi: 10.1002/ejsp.767

Martin, L. L., \& van den Bos, K. (2014). Beyond terror: Towards a paradigm shift in the study of threat and culture. European Review of Social Psychology, 25(1), 32-70. doi: $10.1080 / 10463283.2014 .923144$

Maxfield, M., Greenberg, J., Pyszczynski, T., Weise, D., Kosloff, S., Soenke, M., ... Blatter, J. (2014). Increases in generative concern among older adults following reminders of mortality. International Journal of Aging and Human Development, 79(1), 1-21. doi: 10.2190/AG.79.1.a

Maxfield, M., Pyszczynski, T., Greenberg, J., Pepin, R., \& Davis, H. P. (2012). The moderating role of executive functioning in older adults' responses to a reminder of mortality. Psychology and Aging, 27(1), 256-263. doi: 10.1037/a0023902

Maxfield, M., Pyszczynski, T., Kluck, B., Cox, C. R., Greenberg, J., Solomon, S., \& Weise, D. (2007). Age-related differences in responses to thoughts of one's own death: Mortality salience and judgments of moral transgressions. Psychology and Aging, 22(2), 341-353. doi: 10.1037/0882-7974.22.2.341

McGregor, H. A., Greenberg, J., Arndt, J., Lieberman, J. D., Solomon, S., Simon, L., \& Pyszczynski, T. (1998). Terror Management and aggression: Evidence that mortality salience motivates aggression against worldview-threatening others. Journal of Personality and Social Psychology, 74(3), 590-605. doi: 10.1037/0022-3514.74.3.590 
McShane, B. B., Böckenholt, U., \& Hansen, K. T. (2016). Adjusting for publication bias in meta-analysis: An evaluation of selection methods and some cautionary notes. Perspectives on Psychological Science, 11(5), 730-749.

Munafò, M. R., Nosek, B. A., Bishop, D. V., Button, K. S., Chambers, C. D., Du Sert, N. P., ... Ioannidis, J. P. (2017). A manifesto for reproducible science. Nature human behaviour, 1(1), 1-9.

Nagar, M., \& Rabinovitz, S. (2015). Smoke your troubles away: Exploring the effects of death cognitions on cannabis craving and consumption. Journal of Psychoactive Drugs, 47(2), 91-99. doi: 10.1080/02791072.2015.1029654

Nosek, B. A., Alter, G., Banks, G. C., Borsboom, D., Bowman, S. D., Breckler, S. J., ... Yarkoni, T. (2015). Promoting an open research culture: Author guidelines for journals could help to promote transparency, openness, and reproducibility. Science, 348(6242), 1422-1425.

(Place: US Publisher: American Assn for the Advancement of Science) doi: $10.1126 /$ science.aab2374

Oyserman, D., Coon, H. M., \& Kemmelmeier, M. (2002). Rethinking individualism and collectivism: evaluation of theoretical assumptions and meta-analyses. Psychological bulletin, 128(1), 3 .

Park, Y. C., \& Choi. (2002). Does imaging one's own death make an individual a good citizen? Korean Journal of Social and Personality Psychology, 16(1), 14.

Park, Y. C., \& Pyszczynski, T. (2015). The effects of meditation and mortality salience on in group bias, self-compassion, and compassion-toward-other. Manuscript in preparation.

Park, Y. C., \& Pyszczynski, T. (2016). Cultural universals and differences in dealing with death. In Denying death (pp. 193-213). Routledge. 
Pashler, H., \& Wagenmakers, E. (2012). Editors introduction to the special section on replicability in psychological science. Perspectives on Psychological Science, 7, 528 530.

Proulx, T., \& Heine, S. J. (2006). Death and black diamonds: Meaning, mortality, and the meaning maintenance model. Psychological Inquiry, 17(4), 309-318. doi: $10.1080 / 10478400701366985$

Pyszczynski, T., Solomon, S., \& Greenberg, J. (2015). Thirty years of terror management theory: From genesis to revelation. In Advances in experimental social psychology (Vol. 52, pp. 1-70). Elsevier.

Rodríguez-Ferreiro, J., Barberia, I., González-Guerra, J., \& Vadillo, M. A. (2019). Are we truly special and unique? A replication of Goldenberg et al.(2001). Royal Society open science, 6(11), 191114.

Rosenblatt, A., Greenberg, J., Solomon, S., Pyszczynski, T., \& Lyon, D. (1989). Evidence for Terror Management Theory: I. The effects of mortality salience on reactions to those who violate or uphold cultural values. Journal of Personality and Social Psychology, 57(4), 681-690. doi: 10.1037/0022-3514.57.4.681

Rosenthal, R. (1979). The file drawer problem and tolerance for null results. Psychological Bulletin, 86(3), 638-641. doi: 10.1037/0033-2909.86.3.638

Sætrevik, B., \& Sjåstad, H. (2019). Failed pre-registered replication of mortality salience effects in traditional and novel measures. Retrieved from https://psyarxiv.com/dkg53/

Schimel, J., Hayes, J., Williams, T., \& Jahrig, J. (2007). Is death really the worm at the core? Converging evidence that worldview threat increases death-thought accessibility. 
Journal of Personality and Social Psychology, 92(5), 789-803. doi: 10.1037/00223514.92.5.789

Schindler, S., Pfattheicher, S., Reinhard, M. A., \& Greenberg, J. (2019). Heroes aren't always so great! Heroic perceptions under mortality salience. Social Influence, 14(3-4), 77-91. Retrieved from https://doi.org/10.1080/15534510.2019.1656668 doi: $10.1080 / 15534510.2019 .1656668$

Schindler, S., Reinhardt, N., \& Reinhard, M.-A. (2021). Defending one's worldview under mortality salience: Testing the validity of an established idea. Journal of Experimental Social Psychology, 93, 104087.

Schumaker, J. F., Barraclough, R. A., \& Vagg, L. M. (1988). Death anxiety in Malaysian and Australian university students. The Journal of social psychology, 128(1), 41-47.

Simmons, J. P., Nelson, L. D., \& Simonsohn, U. (2011). False-positive psychology: Undisclosed flexibility in data collection and analysis allows presenting anything as significant. Psychological science, 22(11), 1359-1366.

Simonsohn, U. (2014). No-way interactions. Retrieved from http://datacolada.org/17 Simonsohn, U. (2015). Small telescopes: Detectability and the evaluation of replication results. Psychological science, 26(5), 559-569.

Simonsohn, U. (2017). p-curve app. Retrieved from http://p-curve.com

Simonsohn, U., Nelson, L. D., \& Simmons, J. P. (2014a). P-curve: A key to the file-drawer. (Vol. 143) (No. 2). Simonsohn, Uri: The Wharton School, University of Pennsylvania, 500 Huntsman Hall, 3730 Walnut Street, Philadelphia, PA, US, 19104, uws@wharton.upenn.edu: American Psychological Association. doi: 10.1037/a0033242 
Simonsohn, U., Nelson, L. D., \& Simmons, J. P. (2014b). P-curve and effect size: Correcting for publication bias using only significant results. Perspectives on Psychological Science, 9(6), 666-681. (PMID: 26186117) doi: 10.1177/1745691614553988

Simonsohn, U., Simmons, J. P., \& Nelson, L. D. (2015). Better p-curves: Making p-curve analysis more robust to errors, fraud, and ambitious p-hacking, a reply to Ulrich and Miller (2015). (Vol. 144) (No. 6). Simonsohn, Uri: Wharton School, University of Pennsylvania, 3730 Walnut Street, 500 Huntsman, Philadelphia, PA, US, 19066, uws@wharton.upenn.ed: American Psychological Association.doi:

\section{$10.1037 / x g e 0000104$}

Simonsohn, U., Nelson, L. D., \& Simmons, J. P. (2015). Official user-guide to the $p$ curve [Computer software manual]. Retrieved from http://p-curve.com/guide.pdf

Solomon, S., Greenberg, J., \& Pyszczynski, T. (1991). A Terror Managment Theory of social behavior. Advances in Experimental Social Psychology, 24, 93-159.

Stanley, T. D., \& Doucouliagos, H. (2014). Meta-regression approximations to reduce publication selection bias. Research Synthesis Methods, 5(1), 60-78.

Steegen, S., Tuerlinckx, F., Gelman, A., \& Vanpaemel, W. (2016). Increasing transparency through a multiverse analysis. Perspectives on Psychological Science, 11(5), 702-712. (PMID: 27694465) doi: 10.1177/1745691616658637

Ulrich, R., \& Miller, J. (2015). P-hacking by post hoc selection with multiple opportunities: Detectability by skewness test?: Comment on Simonsohn, Nelson, and Simmons (2014). Journal of Experimental Psychology: General, 144(6), 1137-1145. doi: $10.1037 / x g e 0000086$ 
Vail, K. E., Arndt, J., Motyl, M., \& Pyszczynski, T. (2012). The aftermath of destruction: Images of destroyed buildings increase support for war, dogmatism, and death thought accessibility. Journal of Experimental Social Psychology, 48(5), 1069-1081. doi: 10.1016/j.jesp.2012.05.004

Vail, K. E., Courtney, E., \& Arndt, J. (2019). The influence of existential threat and tolerance salience on anti-Islamic attitudes in American politics. Political Psychology, 40(5), 1143-1162.

Van Den Bos, K. D. (2001). Reactions to perceived fairness: The impact of mortality salience and self-esteem on ratings of negative affect. Social Justice Research, 14(1), 1-23. doi: 10.1023/a:1012501506803

Van Aert, R. C., Wicherts, J. M., \& van Assen, M. A. (2016). Conducting meta-analyses based on p values: Reservations and recommendations for applying $\mathrm{p}$-uniform and $\mathrm{p}$-curve. Perspectives on Psychological Science, 11(5), 713-729.

Van Assen, M. A., Van Aert, R., \& Wicherts, J. M. (2015). Meta-analysis using effect size distributions of only statistically significant studies. Psychological methods, 20(3), 293.

Vohs, K. D., Schmeichel, B. J., Lohmann, S., Gronau, Q. F., Finley, A. J., Ainsworth, S. E., ... Albarracn, D. (2021). A multisite preregistered paradigmatic test of the ego-depletion effect. Psychological Science, 32(10), 1566-1581. Retrieved from https://doi.org/10.1177/0956797621989733 (PMID: 34520296) doi:

\section{$10.1177 / 0956797621989733$}

Wagenmakers, E.-J., Beek, T., Dijkhoff, L., Gronau, Q. F., Acosta, A., R. B. Adams, J., ... Zwaan, R. A. (2016). Registered replication report: Strack, Martin, \& Stepper (1988). Perspectives on Psychological Science, 11(6), 917-928. Retrieved from 
https://doi.org/10.1177/1745691616674458 (PMID: 27784749) doi:

\section{$10.1177 / 1745691616674458$}

Wagenmakers, E.-J., Wetzels, R., Borsboom, D., \& van der Maas, H. L. J. (2011). Why psychologists must change the way they analyze their data: The case of psi: Comment on Bem (2011). Journal of Personality and Social Psychology, 100(3), 426-432. (Place: US Publisher: American Psychological Association) doi: 10.1037/a0022790

Wicherts, J. M., Veldkamp, C. L., Augusteijn, H. E., Bakker, M., Van Aert, R., \& Van Assen, M. A. (2016). Degrees of freedom in planning, running, analyzing, and reporting psychological studies: A checklist to avoid p-hacking. Frontiers in psychology, 7, 1832.

Yen, C.-L., \& Cheng, C.-P. (2010). Terror management among Taiwanese: Worldview defence or resigning to fate? Asian Journal of Social Psychology, 13(3), 185-194. doi: 10.1111/j.1467-839X.2010.01328.X

Yen, C.-L., \& Cheng, C.-P. (2013). Researcher effects on mortality salience research: A metaanalytic moderator analysis. Death studies, 37(7), 636-652. 
Figure 1

Illustrations of potential p-curves generated by 100,000 simulated experiments with $N=87$

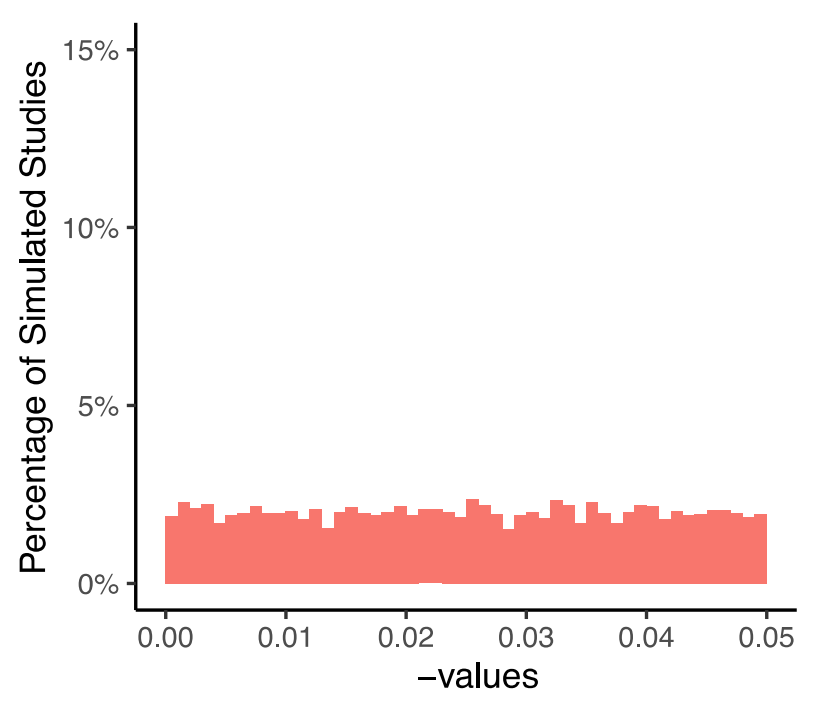

(a) No evidential value

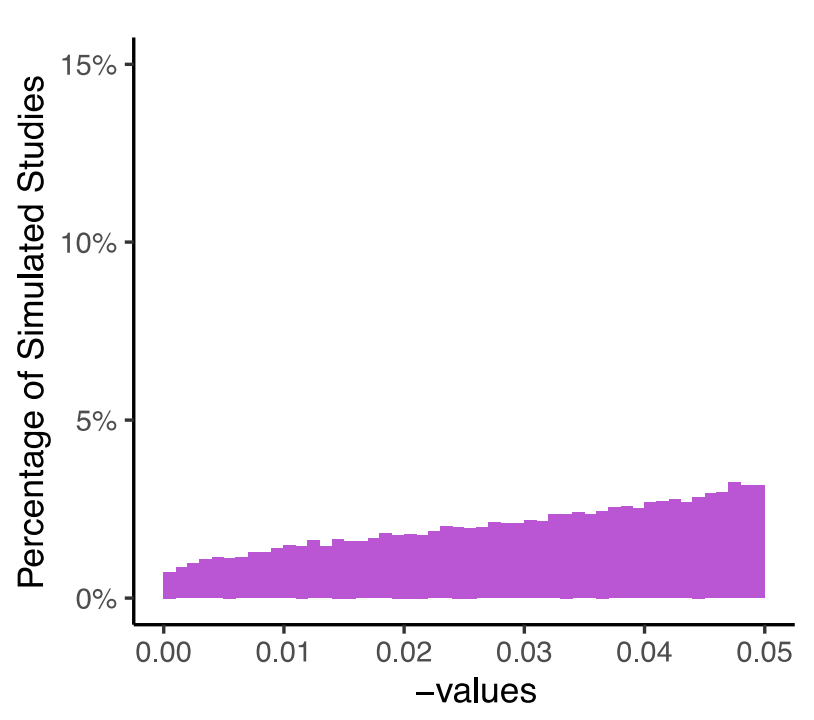

(c) p-hacked

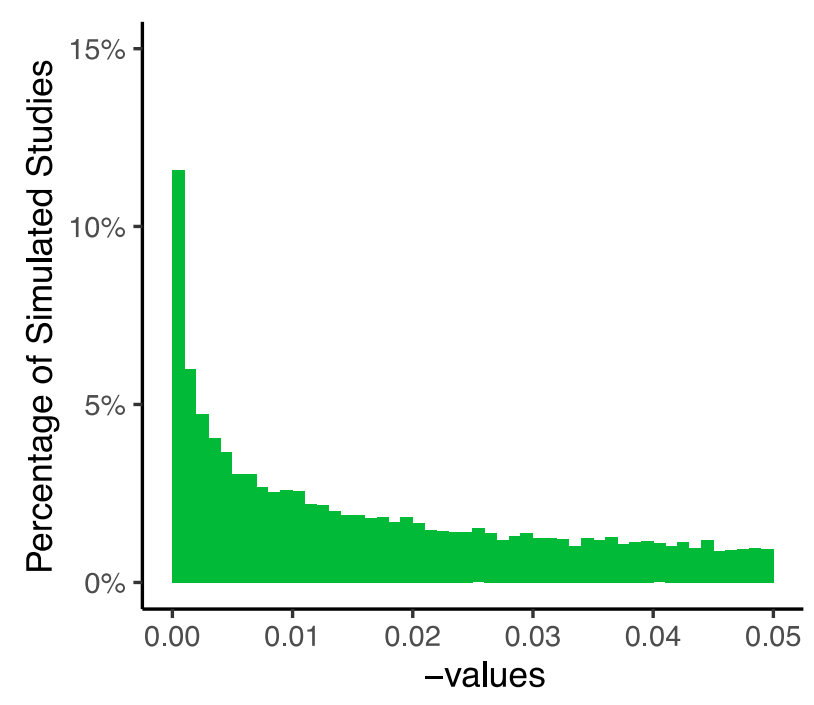

(b) Some evidential value

Note. (a) no population effect; (b) $33 \%$ power, and (c) no population effect with $p$-hacking. $R$ code for $p$-hacking simulation is adapted from Simonsohn (2015). 
Figure 2

Flowchart of the application of exclusion and inclusion criteria

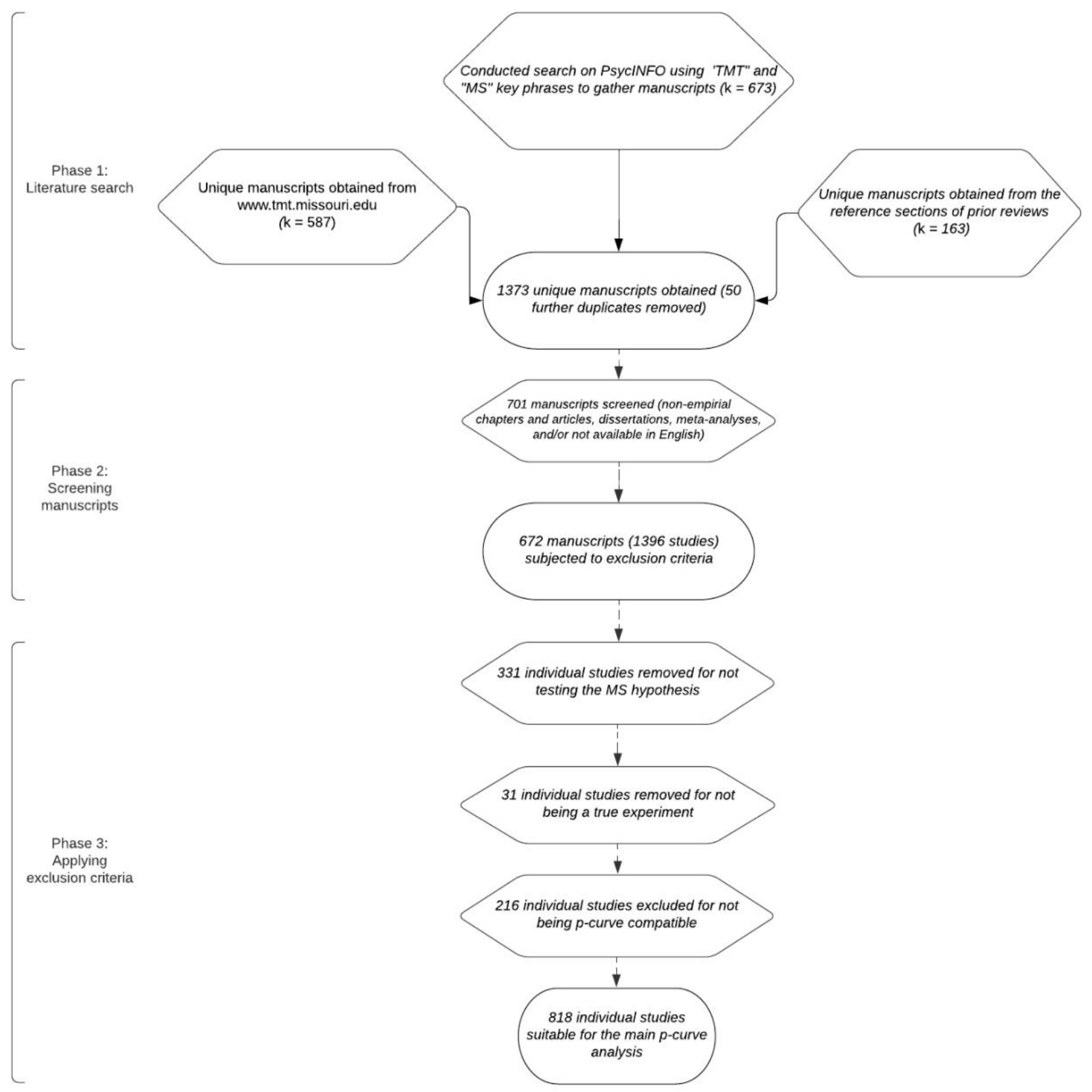


Figure 3

$P$-curve of the entire universe of studies $(K=818)$

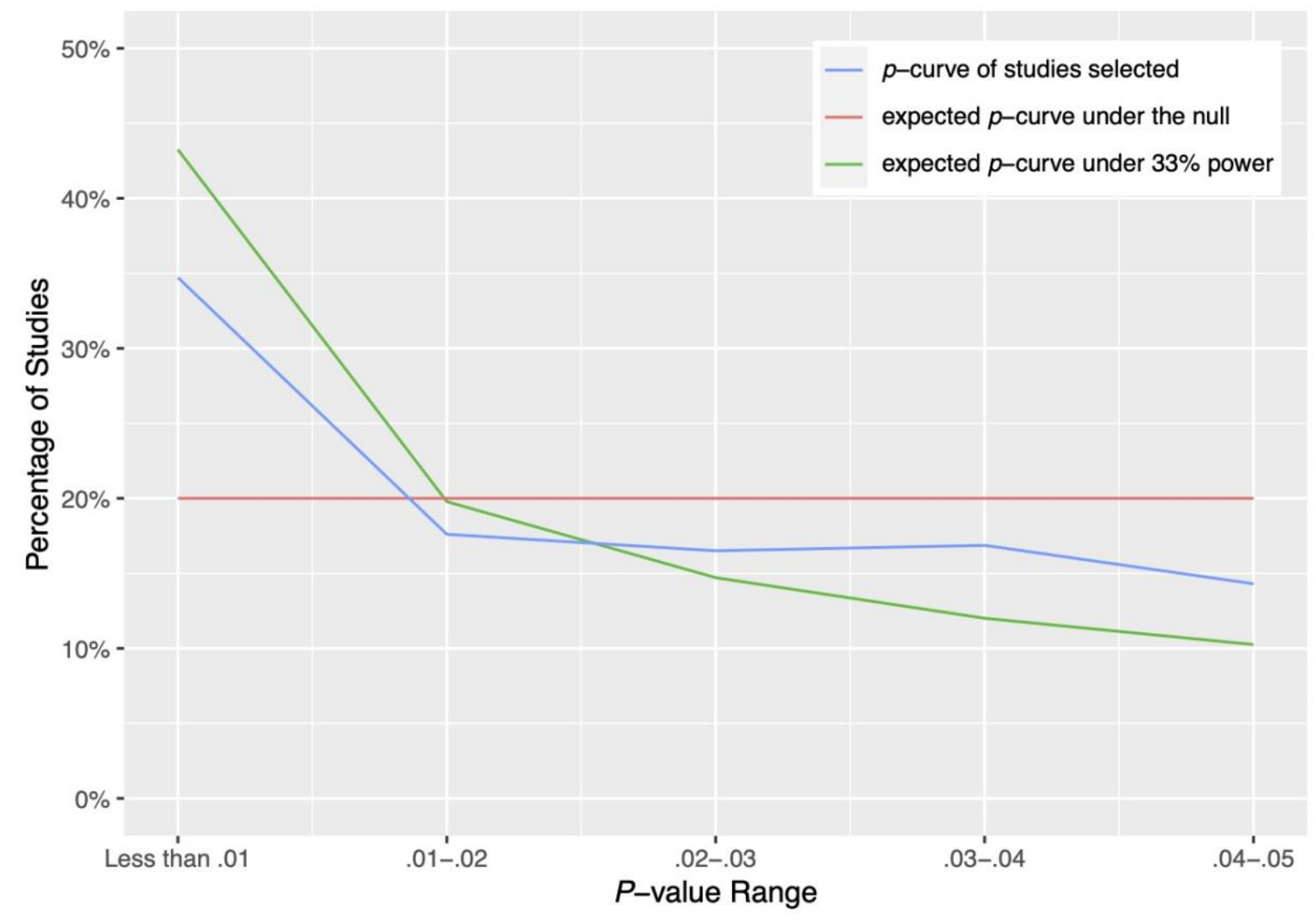


Figure 4

P-curve estimates of average power of the studies by year

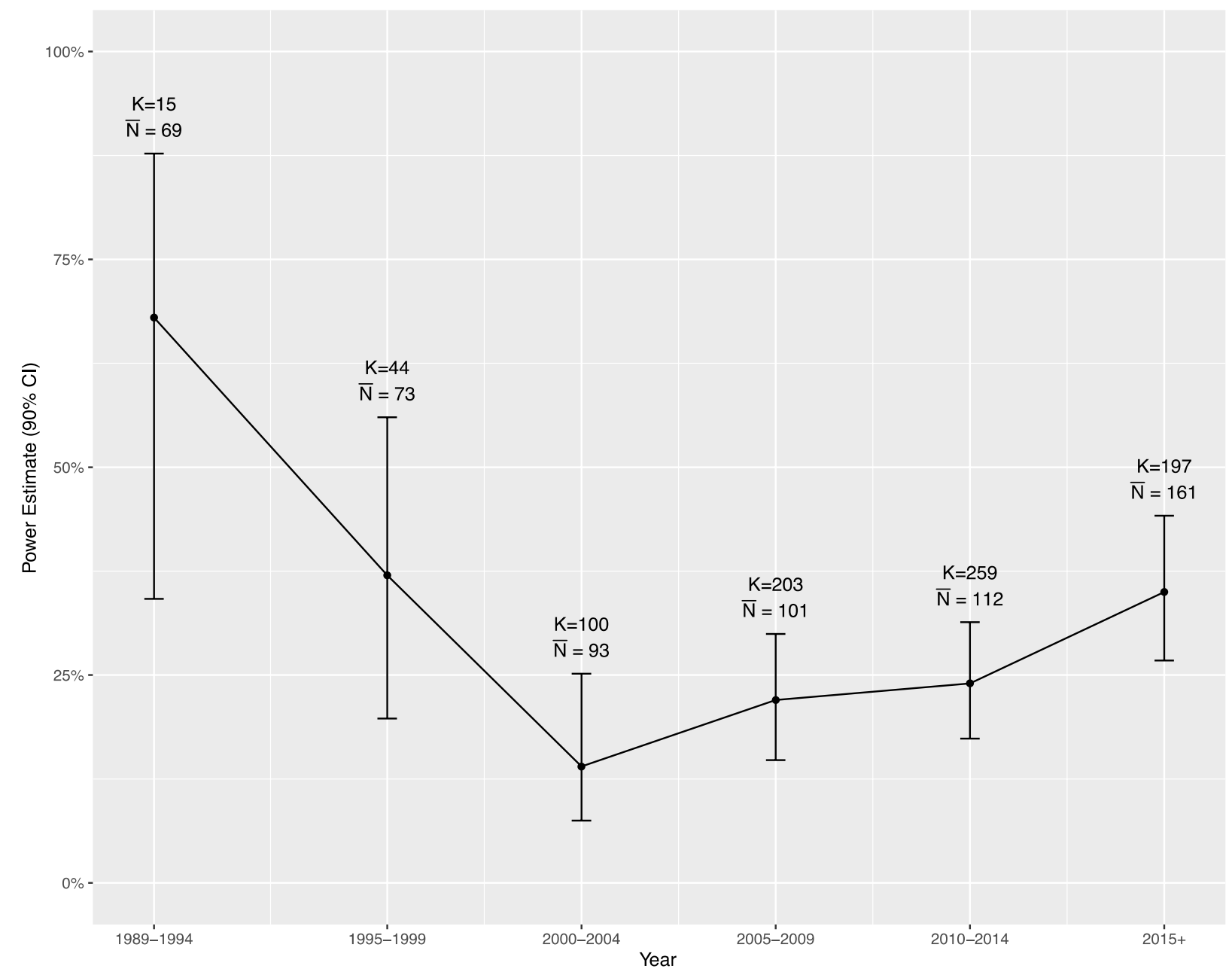

Note. The error bar indicates the lower and upper bounds of the $90 \%$ confidence interval. $\mathrm{K}$ is the number of studies included in the estimate, and $\bar{N}$ is the average sample size of those studies. 
Table 1

Moderators of the effect

\begin{tabular}{|c|c|}
\hline Moderator & Subgroup \\
\hline \multirow[t]{2}{*}{ Main effect or interaction } & Main effect \\
\hline & Interaction \\
\hline \multirow[t]{2}{*}{ Experimental manipulation } & Mortality Attitudes Perception Survey \\
\hline & Other \\
\hline \multirow[t]{2}{*}{ Control condition } & Dental pain \\
\hline & Other \\
\hline \multirow{7}{*}{ Dependent variable type* } & Affect or emotion \\
\hline & Attitudes and beliefs \\
\hline & Behavior \\
\hline & Desire \\
\hline & Cognition \\
\hline & Neural or physiological change \\
\hline & Prosociality \\
\hline \multirow[t]{4}{*}{ Number of delay tasks* } & No delays \\
\hline & One \\
\hline & Two or more \\
\hline & More than zero \\
\hline \multirow[t]{5}{*}{ Interaction type } & Individual difference (trait) \\
\hline & Individual difference (attitude) \\
\hline & Gender \\
\hline & Feature of the task (manipulation) \\
\hline & Feature of the task (attitude target) \\
\hline \multirow[t]{4}{*}{ Country of origin* } & US \\
\hline & Europe and Middle East \\
\hline & East Asia \\
\hline & Canada and Australia \\
\hline \multirow[t]{2}{*}{ Participants* } & College students only \\
\hline & Other \\
\hline \multirow[t]{2}{*}{ Year* } & Before 2011 \\
\hline & After 2011 \\
\hline
\end{tabular}

Note. For Interaction type, variables that do not relate to individual differences or gender are features of the task, changing either the nature of either the manipulation, or attitude target. Preregistered moderators are indicated with*. 
Table 2

Overall and subgroup p-curve results

\begin{tabular}{|c|c|c|c|c|c|c|c|c|c|c|c|c|}
\hline \multirow[t]{2}{*}{ Sample } & \multirow[b]{2}{*}{$\mathrm{K}$} & \multicolumn{5}{|c|}{ Right Skewness test } & \multicolumn{3}{|c|}{ Flatness (33\% Power) Tes } & \multicolumn{3}{|c|}{ Power and $90 \% \mathrm{CI}$} \\
\hline & & Average & $Z_{\text {full }}$ & $p_{\text {full }}$ & $Z_{\text {half }}$ & $p_{\text {half }}$ & $Z_{\text {full }}$ & $\overline{p_{\text {full }}}$ & $p_{\text {binom }}$ & Power & LB & UB \\
\hline Overall & 818 & 115 & -14.76 & $<.001$ & -14.18 & $<.001$ & -2.74 & .003 & $<.001$ & $26 \%$ & $22.2 \%$ & $30.4 \%$ \\
\hline \multicolumn{13}{|l|}{ Main effect or interaction } \\
\hline Main effect & 191 & 97 & -11.46 & $<.001$ & -12.09 & $<.001$ & 2.53 & .994 & .0249 & $48 \%$ & $38.2 \%$ & $56.2 \%$ \\
\hline Interaction & 608 & 121 & -9.93 & $<.001$ & -8.69 & $<.001$ & -4.78 & $<.001$ & $<.001$ & $19 \%$ & $15.4 \%$ & $23.6 \%$ \\
\hline \multicolumn{13}{|l|}{ Experimental manipulation } \\
\hline MAPS & 599 & 114 & -12.48 & $<.001$ & -11.80 & $<.001$ & -2.49 & .007 & $<.001$ & $26 \%$ & $20.8 \%$ & $30.8 \%$ \\
\hline Other & 219 & 117 & -7.90 & $<.001$ & -7.90 & $<.001$ & -1.19 & .117 & .004 & $27 \%$ & $19.8 \%$ & $35.7 \%$ \\
\hline \multicolumn{13}{|l|}{ Control condition } \\
\hline Dental pain & 251 & 115 & -6.19 & $<.001$ & -4.60 & $<.001$ & -3.28 & .001 & .001 & $19 \%$ & $12.9 \%$ & $25.5 \%$ \\
\hline Other & 567 & 115 & -13.62 & $<.001$ & -13.97 & $<.001$ & -1.11 & .133 & $<.001$ & $30 \%$ & $24.9 \%$ & $35.1 \%$ \\
\hline \multicolumn{13}{|l|}{ Dependent variable type } \\
\hline Affect or emotion & 65 & 103 & -4.43 & $<.001$ & -3.97 & $<.001$ & -0.55 & .292 & .242 & $28 \%$ & $15.4 \%$ & $43.8 \%$ \\
\hline Attitudes and beliefs & 418 & 117 & -11.99 & $<.001$ & -10.87 & $<.001$ & -0.67 & .253 & .001 & $31 \%$ & $25.0 \%$ & $37.1 \%$ \\
\hline Behavior & 82 & 117 & -3.91 & $<.001$ & -3.52 & $<.001$ & -1.60 & .055 & .055 & $21 \%$ & $11.2 \%$ & $33.7 \%$ \\
\hline Cognition & 129 & 117 & -5.40 & $<.001$ & -6.68 & $<.001$ & -1.53 & .064 & $<.001$ & $24 \%$ & $14.9 \%$ & $34.1 \%$ \\
\hline Desire & 88 & 123 & -3.53 & $<.001$ & -2.31 & .011 & -2.09 & .018 & .087 & $18 \%$ & $9.6 \%$ & $29.7 \%$ \\
\hline Neural and physiological & 23 & 86 & -1.15 & 124 & -1.69 & .046 & -1.59 & .056 & .047 & $12 \%$ & $5.0 \%$ & $34.3 \%$ \\
\hline Prosociality & 13 & 103 & -1.69 & .045 & -4.99 & $<.001$ & -0.37 & .357 & .003 & $25 \%$ & $5.0 \%$ & $62.1 \%$ \\
\hline \multicolumn{13}{|l|}{ Number of delay tasks } \\
\hline 0 & 124 & 124 & -5.17 & $<.001$ & -5.51 & $<.001$ & -1.52 & .064 & .009 & $23 \%$ & $14.3 \%$ & $34.2 \%$ \\
\hline 1 & 484 & 107 & -10.35 & $<.001$ & -10.05 & $<.001$ & -3.03 & .001 & $<.001$ & $23 \%$ & $18.2 \%$ & $28.5 \%$ \\
\hline $2+$ & 151 & 126 & -7.80 & $<.001$ & -7.02 & $<.001$ & 0.15 & .562 & .166 & $34 \%$ & $24.5 \%$ & $44.6 \%$ \\
\hline$>0$ & 635 & 111 & -12.84 & $<.001$ & -12.23 & $<.001$ & -2.57 & .005 & $<.001$ & $26 \%$ & $21.2 \%$ & $30.6 \%$ \\
\hline \multicolumn{13}{|l|}{ Interaction type } \\
\hline Individual difference (trait) & 233 & 122 & -5.41 & $<.001$ & -3.51 & .001 & -3.72 & $<.001$ & .002 & $17 \%$ & $11.3 \%$ & $23.3 \%$ \\
\hline Individual difference (attitude) & 86 & 134 & -2.30 & .011 & -0.63 & .266 & -3.12 & .001 & .008 & $12 \%$ & $6.2 \%$ & $21.5 \%$ \\
\hline Gender & 57 & 136 & -4.44 & $<.001$ & -5.22 & $<.001$ & -0.30 & .381 & .024 & $31 \%$ & $15.7 \%$ & $48.1 \%$ \\
\hline $\begin{array}{l}\text { Feature of the task } \\
\text { (manipulation) }\end{array}$ & 150 & 117 & -5.31 & $<.001$ & -7.05 & $<.001$ & -2.03 & .021 & $<.001$ & $21 \%$ & $13.6 \%$ & $30.9 \%$ \\
\hline $\begin{array}{l}\text { Feature of the task (attitude } \\
\text { target) }\end{array}$ & 134 & 129 & -6.00 & $<.001$ & -5.05 & $<.001$ & -1.10 & .135 & .057 & $26 \%$ & $17.2 \%$ & $36.9 \%$ \\
\hline \multicolumn{13}{|l|}{ Country of origin } \\
\hline US & 455 & 114 & -11.09 & $<.001$ & -10.15 & $<.001$ & -1.98 & .024 & $<.001$ & $26 \%$ & $21.1 \%$ & $32.2 \%$ \\
\hline Europe and Middle East & 234 & 118 & -5.52 & $<.001$ & -6.36 & $<.001$ & -3.53 & $<.001$ & $<.001$ & $17 \%$ & $11.6 \%$ & $24.1 \%$ \\
\hline East Asia & 55 & 122 & -7.50 & $<.001$ & -7.29 & $<.001$ & 2.57 & .995 & .439 & $58 \%$ & $42.4 \%$ & $71.6 \%$ \\
\hline Canada and Australia & 62 & 100 & -5.74 & $<.001$ & -4.18 & $<.001$ & 0.64 & .739 & .668 & $39 \%$ & $24.3 \%$ & $55.4 \%$ \\
\hline \multicolumn{13}{|l|}{ Participants } \\
\hline College students only & 653 & 103 & -12.37 & $<.001$ & -12.30 & $<.001$ & -3.12 & .001 & $<.001$ & $24 \%$ & $20.0 \%$ & $28.9 \%$ \\
\hline Other & 156 & 165 & -8.22 & $<.001$ & -7.13 & $<.001$ & 0.28 & .609 & .104 & $35 \%$ & $25.5 \%$ & $44.9 \%$ \\
\hline \multicolumn{13}{|l|}{ Year } \\
\hline Before 2011 & 406 & 95 & -9.49 & $<.001$ & -9.23 & $<.001$ & -2.66 & .004 & $<.001$ & $24 \%$ & $18.1 \%$ & $29.6 \%$ \\
\hline After 2011 & 412 & 135 & -11.38 & $<.001$ & -10.82 & $<.001$ & -1.23 & .110 & $<.001$ & $29 \%$ & $23.1 \%$ & $34.9 \%$ \\
\hline
\end{tabular}

Note. Bolded values do not meet the conditions necessary for the presence of adequate evidential value. For

the flatness test, all $p_{\text {half }}$ values were $>.999$ and are omitted from the table. 
Table 3

Estimating the effect size for the universe of studies and subgroups

\begin{tabular}{|c|c|c|c|c|c|c|}
\hline \multicolumn{7}{|c|}{ Corresponding Effect Size (d) } \\
\hline Moderator & Subgroup & Average $\mathrm{N}$ & Power & 2 cells & 4 cells & 8 cells \\
\hline Overall & & 115 & 0.26 & 0.25 & 0.35 & 0.51 \\
\hline \multirow[t]{2}{*}{ Main effect or interaction } & Main effect & 97 & 0.48 & 0.39 & 0.56 & 0.81 \\
\hline & Interaction & 121 & 0.19 & - & 0.28 & 0.41 \\
\hline \multirow[t]{2}{*}{ Experimental Manipulation } & Mortality Attitudes Perception Survey & 114 & 0.26 & 0.25 & 0.35 & 0.51 \\
\hline & Other & 117 & 0.27 & 0.25 & 0.36 & 0.52 \\
\hline \multirow[t]{2}{*}{ Control Condition } & Dental Pain & 115 & 0.19 & 0.20 & 0.29 & 0.42 \\
\hline & Other & 115 & 0.30 & 0.27 & 0.39 & 0.55 \\
\hline \multirow[t]{7}{*}{ Dependent variable type } & Affect or emotion & 103 & 0.28 & 0.27 & 0.39 & 0.57 \\
\hline & Attitudes and beliefs & 117 & 0.31 & 0.27 & 0.39 & 0.56 \\
\hline & Behavior & 117 & 0.21 & 0.21 & 0.31 & 0.44 \\
\hline & Cognition & 117 & 0.24 & 0.23 & 0.33 & 0.48 \\
\hline & Desire & 123 & 0.18 & 0.19 & 0.27 & 0.39 \\
\hline & Neural and physiological & 86 & 0.12 & 0.17 & 0.24 & 0.35 \\
\hline & Prosociality & 103 & 0.25 & 0.26 & 0.36 & 0.53 \\
\hline \multirow[t]{4}{*}{ Number of delay tasks } & 0 & 124 & 0.23 & 0.22 & 0.31 & 0.45 \\
\hline & 1 & 107 & 0.23 & 0.24 & 0.34 & 0.49 \\
\hline & $2+$ & 126 & 0.34 & 0.28 & 0.40 & 0.57 \\
\hline & $>0$ & 111 & 0.26 & 0.25 & 0.36 & 0.52 \\
\hline \multirow[t]{5}{*}{ Interaction Type } & Individual difference (trait) & 122 & 0.17 & 0.18 & 0.26 & 0.37 \\
\hline & Individual difference (attitude) & 134 & 0.12 & 0.13 & 0.19 & 0.27 \\
\hline & Gender & 136 & 0.31 & 0.25 & 0.36 & 0.52 \\
\hline & Feature of the task (manipulation) & 117 & 0.21 & 0.21 & 0.31 & 0.44 \\
\hline & Feature of the task (attitude target) & 129 & 0.26 & 0.23 & 0.33 & 0.48 \\
\hline \multirow[t]{4}{*}{ Country of origin } & US & 114 & 0.26 & 0.25 & 0.35 & 0.51 \\
\hline & Europe and Middle East & 118 & 0.17 & 0.19 & 0.26 & 0.38 \\
\hline & East Asia & 122 & 0.58 & 0.39 & 0.56 & 0.81 \\
\hline & Canada and Australia & 100 & 0.39 & 0.34 & 0.48 & 0.70 \\
\hline \multirow[t]{2}{*}{ Participants } & College students & 103 & 0.24 & 0.25 & 0.36 & 0.51 \\
\hline & Other & 165 & 0.35 & 0.25 & 0.35 & 0.50 \\
\hline \multirow[t]{2}{*}{ Year } & Before 2011 & 95 & 0.24 & 0.26 & 0.37 & 0.54 \\
\hline & After 2011 & 135 & 0.29 & 0.24 & 0.35 & 0.50 \\
\hline
\end{tabular}

Note. Effect sizes corresponding to the $p$-curve power estimate and average sample size given study designs with 2 cells, 4 cells, and 8 cells. Bolded values denote $p$-curves corresponding to power significantly below $33 \%$. Due to the generally low evidential values, these estimates may not be reliable. 